\title{
Plasmonic-perovskite solar cells, light emitters, and sensors
}

\author{
Bin Ai $\oplus^{1,2,3}$, Ziwei Fan and Zi Jing Wong ${ }^{1,4 凶}$
}

\begin{abstract}
The field of plasmonics explores the interaction between light and metallic micro/nanostructures and films. The collective oscillation of free electrons on metallic surfaces enables subwavelength optical confinement and enhanced light-matter interactions. In optoelectronics, perovskite materials are particularly attractive due to their excellent absorption, emission, and carrier transport properties, which lead to the improved performance of solar cells, lightemitting diodes (LEDs), lasers, photodetectors, and sensors. When perovskite materials are coupled with plasmonic structures, the device performance significantly improves owing to strong near-field and far-field optical enhancements, as well as the plasmoelectric effect. Here, we review recent theoretical and experimental works on plasmonic perovskite solar cells, light emitters, and sensors. The underlying physical mechanisms, design routes, device performances, and optimization strategies are summarized. This review also lays out challenges and future directions for the plasmonic perovskite research field toward next-generation optoelectronic technologies.
\end{abstract}

\section{Introduction}

Halide perovskite materials have an $\mathrm{ABX}_{3}$ chemical formula where A can be an organic or an inorganic cation (e.g., methylammonium (MA, $\mathrm{CH}_{3} \mathrm{NH}_{3}{ }^{+}$), formamidinium (FA, $\mathrm{CH}\left(\mathrm{NH}_{2}\right)_{2}{ }^{+}$), $\mathrm{Cs}^{+}, \mathrm{Rb}^{+}$, or their mixtures), $\mathrm{B}$ is a divalent cation (e.g., $\mathrm{Pb}^{2+}$ or $\mathrm{Sn}^{2+}$ ) and $\mathrm{X}$ is a halide anion (e.g., $\mathrm{I}^{-}, \mathrm{Br}^{-}, \mathrm{Cl}^{-}$or their combinations). The lattice arrangement of perovskite is shown in Fig. 1a, where the larger atom A sits at the center of a cube, B occupies the corners of the cube, and the smaller atom $\mathrm{X}$ is on the faces of the cube. Halide perovskites have low recombination losses, large bandgap tunability, large absorption coefficients, high defect tolerance, and long charge carrier diffusion lengths and lifetimes ${ }^{1-3}$, all of which lead to efficient absorption, photocarrier extraction, and light emission properties, as shown in Fig. 1b, c. Moreover, halide perovskites can be solution processed at

Correspondence: Zi Jing Wong (zijing@tamu.edu)

'Department of Aerospace Engineering, Texas A\&M University, College Station, TX 77843, USA

${ }^{2}$ School of Microelectronics and Communication Engineering, Chongqing University, 400044 Chongqing, P.R. China

Full list of author information is available at the end of the article low temperatures, which significantly reduces their fabrication cost and complexity ${ }^{4}$. These advantages have led to the emergence of a variety of novel perovskite-based devices in the past decade ${ }^{5,6}$, such as solar cells $(\mathrm{SCs})^{7-10}$, light-emitting diodes (LEDs) ${ }^{11-14}$, lasers ${ }^{15-17}$, photodetectors (PDs) ${ }^{18-20}$, sensors ${ }^{21,22}$, catalyst electrodes ${ }^{23-25}$, field-effect transistors ${ }^{26,27}$, fuel cells ${ }^{28,29}$, memory ${ }^{30,31}$ and spintronic devices ${ }^{32,33}$. However, halide perovskites are prone to phase changes and compositional degradation in the ambient environment. Despite that, the merits and prospects of halide perovskites are still very promising, and there are many passivation, encapsulation, compositional engineering, and novel deposition techniques to enhance halide perovskite stability.

The most prominent application of halide perovskites is as light-absorbing materials in solar cells. Miyasaka and coworkers first applied halide perovskite materials in dyesensitized solar cells in $2009^{34}$. In 2012, Nam Gyu Park's group revealed the great potential of perovskites by reporting a lead iodide perovskite solar cell (PSC) with a power conversion efficiency (PCE) above $9 \%{ }^{35}$. Since then, PSCs have experienced rapid and continuous breakthroughs in regard to their PCEs. The larger

\section{(c) The Author(s) 2022}

(c) (i) Open Access This article is licensed under a Creative Commons Attribution 4.0 International License, which permits use, sharing, adaptation, distribution and reproduction cc) in any medium or format, as long as you give appropriate credit to the original author(s) and the source, provide a link to the Creative Commons license, and indicate if changes were made. The images or other third party material in this article are included in the article's Creative Commons license, unless indicated otherwise in a credit line to the material. If material is not included in the article's Creative Commons license and your intended use is not permitted by statutory regulation or exceeds the permitted use, you will need to obtain permission directly from the copyright holder. To view a copy of this license, visit http://creativecommons.org/licenses/by/4.0/. 
a

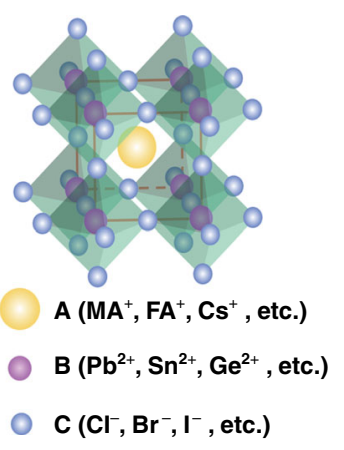

d

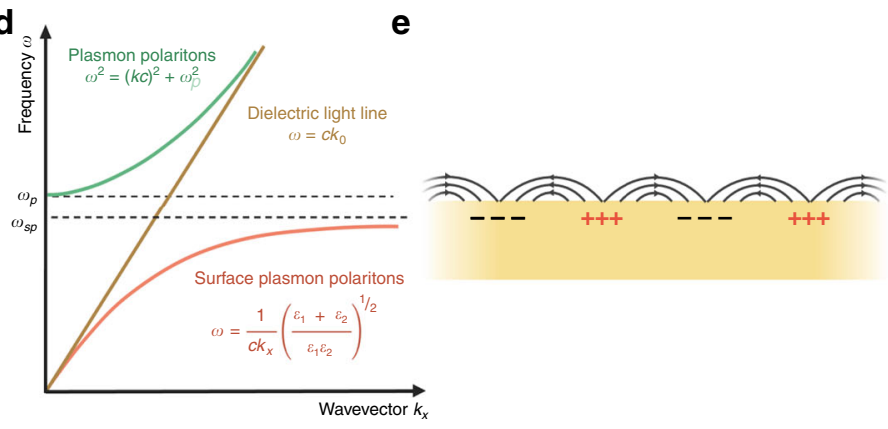

c

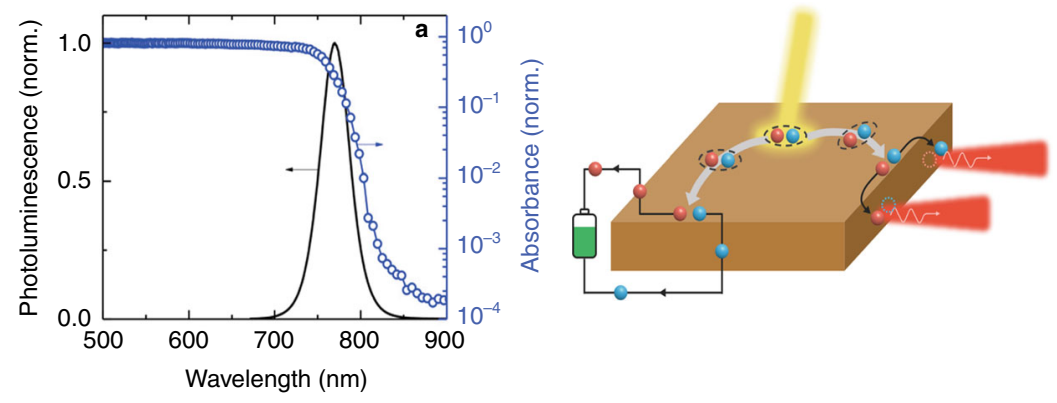

f

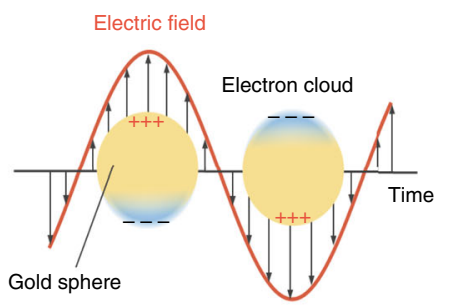

Fig. 1 Outline of perovskites and plasmonics. a Structure of a halide perovskite unit cell. b Photoluminescence spectrum (left axis) and absorption spectrum as measured by photothermal deflection spectroscopy (PDS, right axis) of a $\left(\mathrm{Cs}_{0.06} \mathrm{MA}_{0.15} \mathrm{FA} \mathrm{A}_{0.79}\right) \mathrm{Pb}\left(\mathrm{I}_{0.85} \mathrm{Br}_{0.15}\right)_{3}$ thin film. Adapted with permission $^{213}$. Copyright 2019, Wiley. c Schematic of the process of photocarrier extraction and light emission. $\mathbf{d}$ Photon dispersion in the bulk of a metal and surface plasmon polariton dispersion on the surface of the same metal and a dielectric. e Surface plasmon polariton (SPP) and (f) localized surface plasmon resonance modes (LSPRs).

bandgaps of halide perovskites compared with traditional photovoltaic materials such as $\mathrm{Si}$ and GaAs also enable them to form tandem solar cells with lower-bandgap photovoltaic materials to attain even higher PCEs. Furthermore, PSCs offer additional attributes such as their semitransparency, light weight, and flexibility. The PCEs of PSCs have now exceeded 25\%, which is only slightly lower than the $27.6 \%$ PCE of the best single-crystalline silicon solar cells ${ }^{36}$. In addition to improving material quality and stability of perovskites, new design strategies to further improve the PCE are critical for nextgeneration photovoltaics (PVs).

Halide perovskites have also been used as light-emitting materials in LEDs and lasers. In perovskites, holes and electrons are confined into inorganic $\left[\mathrm{PbX}_{6}\right]^{4-}$ octahedral networks, leading to strong Coulomb interactions and excitonic effects. They also have the advantages of a high quantum yield (QY), narrow band emission, and wide color tunability across the entire visible and infrared region, which makes them a promising material to use in light-emitting devices. $\mathrm{CsPbBr}_{3}$ perovskite nanocrystals (NCs) exhibit a high photoluminescence quantum yield (PLQY) of $\sim 97 \%{ }^{37}$. Moreover, the emission color can be facilely tuned in the visible range (blue to red) by varying the halide anion $\left(\mathrm{Cl}^{-}, \mathrm{Br}^{-}\right.$, or I- $)$, showing the potential of perovskites in white LEDs ${ }^{38,39}$. Recently, the efficiency of LEDs reached $108 \mathrm{~cd} / \mathrm{A}$ (external quantum efficiency (EQE) of 23.4\%) and was further increased to $205 \mathrm{~cd} / \mathrm{A}$ (EQE of 45.5\%) with a hemispherical lens ${ }^{40}$. Compared with those of mature technologies such as organic LEDs $(\text { EQE of } 25 \%)^{41}$ and inorganic quantum dot LEDs (20.5\%), this is a high efficiency ${ }^{42}$. Similarly, low-threshold lasers can also be achieved using halide perovskites as the gain materials. The optical gain coefficients of lead-halide perovskites can be comparable to those of conventional bulk semiconductors, such as $\mathrm{GaAs}^{43}$. Moreover, the emission wavelengths of lead-halide perovskite lasers can be tuned from ultraviolet to near-infrared (NIR) by controlling the composition ${ }^{44-46}$, filling the green emission gap between III-nitrides and III-phosphides. Various perovskite microlasers have been realized by microplates $^{47}$, micro/nanorods ${ }^{48}$, microdisks ${ }^{49}$, and photonic crystals $^{50,51}$. Recently, continuous wave $(\mathrm{CW})$-pumped $\mathrm{MAPbX}_{3}$ perovskites have been reported, showing the possibility of electrically driven perovskite microlasers ${ }^{52}$. Perovskite lasers can also realize unidirectional emissions $^{49}$, mode control ${ }^{53}$, and high-density laser arrays ${ }^{54,55}$. Despite rapid progress, there is considerable room to 
improve the crystal quality and optical gain, as well as the $\mathrm{EQE}$, lasing threshold, and emission lifetime.

Benefiting from the strong light absorption of halide perovskites, new applications have been found in regard to sensing and signal detection ${ }^{56-60}$. Novel perovskite detectors and sensors exhibit broad detection ranges, low detection limits, and fast response speeds. Xia et al. first reported a halide perovskite $\left(\mathrm{CH}_{3} \mathrm{NH}_{3} \mathrm{PbI}_{3}\right)$ photodetector in $2014^{61}$. The responsivity and response time were $0.49 \mu \mathrm{A} \mathrm{W}^{-1}$ and $0.02 \mathrm{~s}$, respectively. Four years later, halide perovskite PDs achieved a responsivity of $5.6 \times 10^{8}$ $\mathrm{A} \mathrm{W}^{-1}$, a detectivity of $2.8 \times 10^{16}$ Jones, and a linear dynamic range of $92 \mathrm{~dB}^{62}$. Notably, every figure of merit is comparable with conventional complementary metal oxide semiconductor technologies; however, perovskites hold the advantages of a lower material cost and potentially greater flexibility. Halide perovskites have been used for different sensing applications, such as detecting volatile organic compounds ${ }^{63}$, liquid/solid compounds ${ }^{64}, \mathrm{pH}$ values/temperatures ${ }^{65}$, and pressures ${ }^{66}$. As researchers continue to expand the applicability of perovskite sensors, there is also a need to develop more compact sensing interfaces with a stronger light detection capability.

In the past decade, perovskite optoelectronic studies have mostly focused on enhancing the intrinsic properties of perovskite materials (e.g., via crystal quality optimization $^{7}$, composition optimization ${ }^{9}$, and surface passiva$\left.\operatorname{tion}^{67,68}\right)$. However, there is always a ceiling for intrinsic property improvements, which calls for a new strategy to boost the performance of perovskite devices beyond the intrinsic limits of perovskites. This alternative strategy could be plasmonics, which can both enhance light absorption and modify electronic behavior ${ }^{69-72}$. The field of plasmonics relies on the collective oscillations of electrons excited by electromagnetic radiation at a metal-dielectric interface, which gives rise to the term surface plasmon resonance (SPR). SPR enables large wavevectors and thus field confinement (Fig. 1d $)^{73-75}$. There are two types of plasmonic modes, propagating surface plasmon polaritons (SPPs) (Fig. 1e) and localized surface plasmon resonances (LSPRs) (Fig. 1f), and these are usually excited on metal films and metal nanoparticles (NPs), respectively. Light can be confined to the nanometer-deep subwavelength level in the proximity of a plasmonic nanostructure and induces strong electric field ( $E$-field) enhancement. In addition to near-field enhancement, plasmonics can also result in strong far-field scattering, excitation of hot electrons, and localized photothermal heating. These effects are highly dependent on the structural morphology of metals, the permittivity of the surrounding dielectrics, and their light polarization and wavelength. In conventional optoelectronics (without perovskites), plasmonic effects have been intensively studied and proven to be effective. The ability of plasmonics to concentrate light at the nanoscale and enhance light-matter interaction holds the key to significantly improving the performance of perovskite optoelectronic devices.

Here, we review the recent works of plasmonic and perovskite-based solar cells, LEDs, lasers, sensors and other applications to provide a more comprehensive summary of plasmonic implementation and effects on halide perovskite devices. Both theoretical and experimental efforts will be covered, highlighting conceptual advances and key breakthroughs. The outline is as follows.

The first section (Plasmonic-perovskite solar cells) classifies the studies in plasmonic PSCs into NP-assisted and plasmonic film-assisted PSCs according to the structure of metal additives and the plasmonic modes. As NP-assisted PSCs have been widely studied both theoretically and experimentally, they are split into two separate subsections of simulation and experimentation to clearly distinguish between the theoretical predictions and attained experimental results. The effect of the configuration, geometry, size, and concentration of plasmonic NPs on solar cell performance is discussed. The second section (Plasmonic-perovskite light emitters) is focused on exploiting the plasmonic nanostructures in perovskite light emitters, including enhanced spontaneous emission and lasers. The third section (Plasmonic-perovskite sensors) discusses how plasmonics and perovskites are combined for sensors such as PDs and optical sensors. Other representative plasmonic-perovskite applications are presented in the fourth section. Regarding plasmonic-perovskite emitters, sensors, and other applications, specific simulation studies are rarer; thus, we introduce them together with experimental works. Finally, we summarize future avenues and challenges for plasmonic-perovskite applications.

\section{Plasmonic-perovskite solar cells}

Plasmonic effects in SCs can be invoked by LSPRs and SPPs. LSPRs are mainly found in metal NPs and are dependent on the size, shape, material, and surrounding environment of the metal NPs. LSPRs lead to enhanced local $E$-fields and optical extinction. Maximum field enhancement is usually found at regions closest to the NP surface, and the field decreases exponentially within $\sim 20-30 \mathrm{~nm}$ away from the surface. On the other hand, SPPs are excited by coupling light waves with electron oscillations on metal films. Incident light can therefore be converted into LSPRs and/or SPPs at different layers within the PSCs to attain higher light-harvesting efficiency. Plasmonic PSCs will be discussed in the subsections of NPs and plasmonic films.

\section{NP-assisted perovskite solar cells}

PSCs are typically composed of an electron transport layer (ETL, e.g., $\mathrm{TiO}_{2}$ or phenyl- $\mathrm{C}_{61}$-butyric acid methyl 


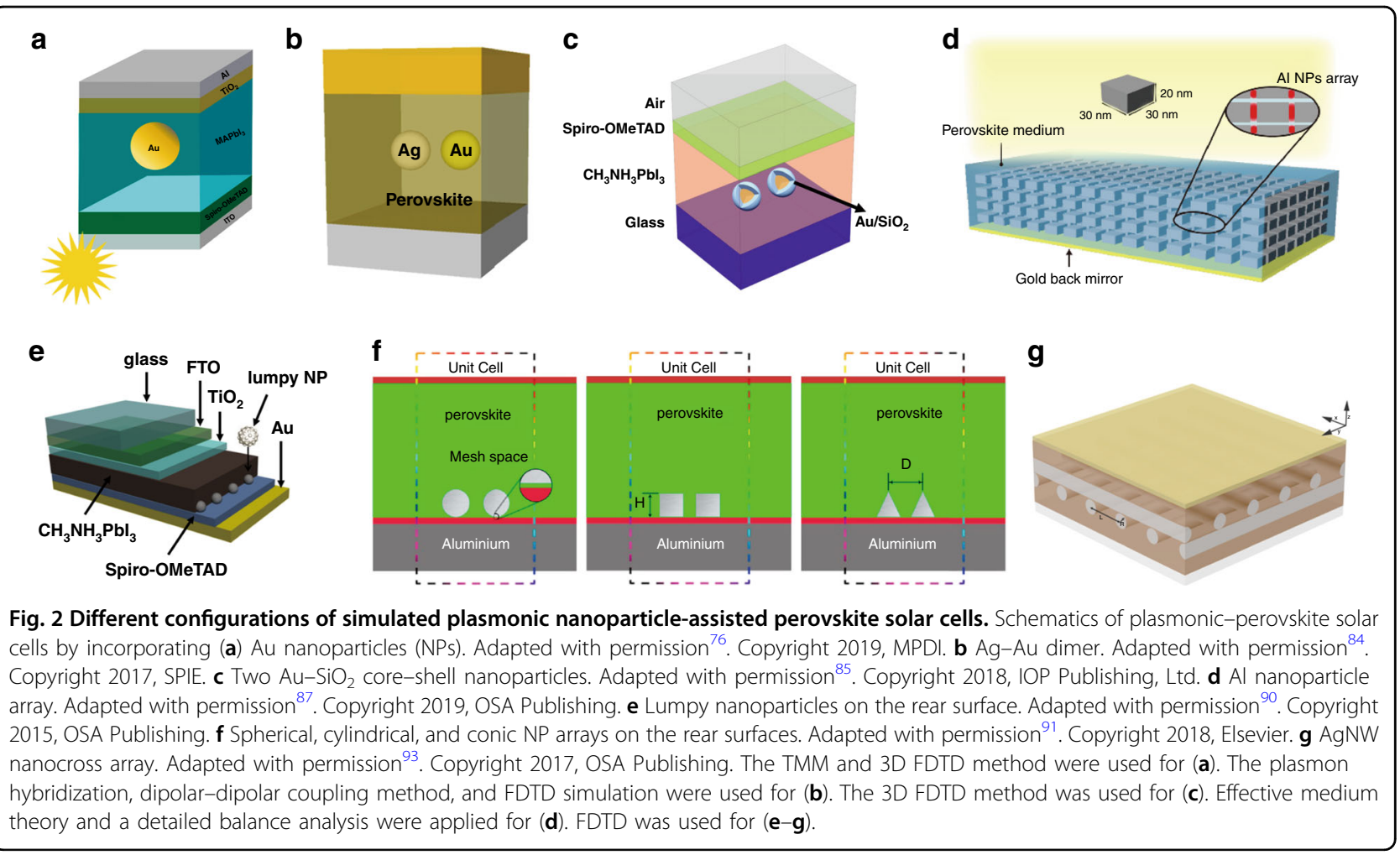

ester (PCBM)), an active layer (halide perovskite), and a hole transport layer (HTL, e.g., poly(3,4-ethylenedioxythiophene)-poly(styrenesulfonate) (PEDOT:PSS) or spiro-OMeTAD) sandwiched by a transparent electrode (fluorine-doped tin oxide (FTO) or indium-doped tin oxide (ITO)) and a metal electrode ( $\mathrm{Au}, \mathrm{Ag}$, or $\mathrm{Al}$ ). In principle, NPs can be incorporated in ETLs, perovskite layers, and HTLs to alter light absorption. It is imperative to systematically summarize PV performances and clarify the underlying enhancement mechanisms and effects of the NP geometry, size, concentration, position, and composition. In the following, we first introduce the advances in simulation and then the experimental results.

\section{Simulations of NP-assisted perovskite solar cells}

Spherical NPs are used most commonly in PSCs and are usually studied by embedding them in the perovskite layer; one example is shown in Fig. $2 \mathrm{a}^{76,77}$. Chang et al. assessed the optical effects of $\mathrm{Cu}$ NPs embedded in a perovskite film by the transfer matrix method (TMM) and three-dimensional finite difference time domain (3D FDTD) method $^{78}$. The thickness of the perovskite and the gap size between $\mathrm{Cu}$ NPs were varied, which led to the conclusions that $\mathrm{Cu}$ NPs could reduce the perovskite thickness from $400 \mathrm{~nm}$ to $300 \mathrm{~nm}$ while maintaining the absorption strength and that the absorbance at $\lambda=$ $350-760 \mathrm{~nm}$ increased by $1.7 \%$ when the gap size was $30 \mathrm{~nm}$ (NP diameter was $70 \mathrm{~nm}$ ). The trapping of incident light between the $\mathrm{Cu}$ NPs and perovskite led to a higher absorption efficiency. Palacios et al. presented an FDTDbased PSC model with Au NPs to analyze the effect of size, concentration, and location in the perovskite film ${ }^{79}$. Solar absorption could be enhanced by $\sim 10 \%$ when the thickness of the perovskite films was $200 \mathrm{~nm}$ and the radius of the spheres was $60 \mathrm{~nm}$. The enhancement was $\sim 6 \%$ when the thickness of the perovskite films was $300 \mathrm{~nm}$ and the radius of the spheres was $90 \mathrm{~nm}$. The enhanced absorption results arose from both the plasmonic near-field and scattering effects. Pathak et al. calculated the optical cross-section of arbitrarily sized and spherical-shaped metal NPs with perovskite by Mie scattering theory ${ }^{80}$. They found that NPs with a radius of $15 \mathrm{~nm}$ and volume concentration of approximately $10 \%$ achieved the highest scattering efficiency. The absorption and scattering efficiencies of Ag NPs with varying sizes, shapes, and polarizations of linearly polarized light were studied using the discrete dipole approximation (DDA) method in the active layer of PSCs ${ }^{81}$. Researchers found that the incorporation of Ag NPs in the active layer of PSCs led to a strong LSPR. Yue et al. proposed a full-wave simulation approach to demonstrate the effects of closely and regularly spaced Ag NPs in the perovskite layer of $\mathrm{PSCs}^{82}$. The infrared absorption of the PSC integrated with Ag NPs was enhanced by $58.2 \%$. Coated and uncoated Ag NPs (radii ranging from 10 to $15 \mathrm{~nm}$ ) were studied to understand the plasmonic interaction with 
perovskite dielectric media ${ }^{83}$. The SPR peaks of the coated Ag NPs could be tuned over a broader range than those of the uncoated Ag NPs.

In addition to works using isolated NPs (in the unit cell), the performance of dimers has been thoroughly studied. Hu et al. discussed the implication of employing random $\mathrm{Ag}-\mathrm{Au}$ heterodimers in $\mathrm{MAPbI}_{3} \mathrm{SCs}$ (Fig. 2b) by plasmon hybridization, the dipolar-dipolar coupling method, and FDTD simulation ${ }^{84}$. The Ag-Au heterodimers provided an enhanced optical field in both the junction (gap) and end areas (no-junction area near the surface of the NPs), while the Au/Ag homodimers only formed an enhanced optical field in the junction area. The absorption was enhanced by $28.15 \%$ for a $150-\mathrm{nm}$ perovskite film embedded with random $\mathrm{Ag}-\mathrm{Au}$ heterodimers (80-nm diameter and 25-nm gap) when compared to a perovskite film without NPs. The absorption enhancement was higher than that of Au homodimers whose absorption enhancement was $10 \%$. The light absorption of the perovskite-embedded heterodimers was higher than that of the homodimers due to the radiation effect in the end area and the strongly enhanced local $E$-fields in the junction area. The performance of core-shell dimer NPs in PSCs was calculated by the 3D FDTD method (Fig. 2c) ${ }^{85}$. The maximum photocurrent of the $\mathrm{Au}-\mathrm{SiO}_{2}$ core-shell dimer with a radius of $60 \mathrm{~nm}$ and a gap distance of $60 \mathrm{~nm}$ was $23.37 \mathrm{~mA} / \mathrm{cm}^{2}$, which was larger than that of the $\mathrm{Au}$ dimer $\left(22.5 \mathrm{~mA} / \mathrm{cm}^{2}\right)$, reference cell $\left(19.8 \mathrm{~mA} / \mathrm{cm}^{2}\right)$, and cell with a single embedded NP $\left(17.9 \mathrm{~mA} / \mathrm{cm}^{2}\right)$. The thin dielectric shell acted as an insulator to prevent charge recombination at the metal-absorber interface, leading to the largest enhancement in PSC performance.

Scaffolds were added to the perovskite for better dispersion of the NPs. Ghahremanirad et al. employed Au NPs in a kesterite mesostructure embedded in perovskite $^{86}$. The plasmonic network and kesterite mesostructure can induce strong near fields in the absorber layer and enhance light absorption. As a result, more light will be confined within the perovskite layer. The absorbance spectrum was broadened, and the EQE of the planar PSC was enhanced by $29 \%$. PSCs with Al NPs in the interstices of perovskite grids (Fig. 2d) were explored by effective medium theory and a detailed balance analysis ${ }^{87}$, showing that the performance of PSCs would be enhanced if the effective refractive index of the perovskites with Al NPs increased.

Several papers have modeled PSCs with plasmonic structures embedded in layers other than the perovskite layer. Hajjiah et al. studied the effect of adding $\mathrm{Au}$ and $\mathrm{Ag}$ NPs to the rear side of a PSC based on $\mathrm{TMM}^{88}$. The resonance wavelength of the NPs was tuned, especially in the wavelength range of red, to enhance the absorption of visible light. Both $\mathrm{Au}$ and $\mathrm{Ag}$ NPs led to a significant enhancement in the short current $\left(J_{\text {sc }}\right)$ when the size of the NPs exceeded $40 \mathrm{~nm}$. Overall, the EQE was enhanced, and the EQE improvement was slightly higher with Ag NPs than with Au NPs. E. Ghahremanirad et al. theoretically demonstrated that the performance of planar PSCs was enhanced when HTL was a hexagonal $\mathrm{NiO}$ nanoprism array with Au NPs distributed in the gaps ${ }^{89}$. The calculations were based on the 3D FDTD method and the finite element method (FEM). The absorptivity of PSCs in the NIR region was enhanced by the Au NPs around the nanoprism array in the HTL. The Au NPs achieved the highest enhancement when the radius was $10 \mathrm{~nm}$. The light-trapping ability of plasmonic nanostructures inside the active layer was increased by the tight coupling of the plasmonic NPs.

In addition to spherical NPs, NPs with various geometries have been examined in the search for better performance. Cai et al. studied the effects of adding lumpy Ag NPs to the rear facet of PSCs by running FDTD simulations (Fig. 2e $)^{90}$. The lumpy NPs consisted of a large Ag core (radius $=100 \mathrm{~nm}$ ) attached to small Ag NPs (radius $=10 \mathrm{~nm}$ ). The lumpy Ag NPs provided the highest power conversion efficiency (PCE) when compared to the devices without NPs and with $\mathrm{Ag}(\mathrm{Au})$ spherical NPs. This enhancement was because the small Ag NPs enhanced the local $E$-field and the scattering was improved by the large Ag NPs over a broadband spectrum (300-900 nm). Pt NPs in triangular, rectangular, and spherical forms were embedded in the perovskite absorber (Fig. 2f) ${ }^{91}$. A finite-sized PSC model with different plasmonic arrays was constructed using the FDTD solutions of Maxwell's equations. The triangular array showed almost the same performance as the rectangular array, decreasing the light reflectance to $6 \%$ and enhancing the current density by $22 \%$ from $39.2 \mathrm{~mA} / \mathrm{cm}^{2}$ (without any other NPs) to $47.9 \mathrm{~mA} / \mathrm{cm}^{2}$. Notably, the use of NPs in the active layer prevented other layers from parasitic absorption. Furthermore, cylinders ${ }^{92}$ and nanowires $(\mathrm{NWs})^{93}$ (Fig. 2g) of different sizes, materials, and arrangements have also been studied and have achieved significant absorption and PCE enhancements.

Abdelraouf et al. calculated the effects of various nanostructured antireflective coatings, including spheres, cylinders, cones, cubes, and cylindrical shells, as well as their coupling, on the efficiency of PSCs based on Mie theory and a 3D FEM optical and electrical model ${ }^{94}$. It was found that the cones and cylindrical shells were not recommended due to the existence of various dipole modes that increased light losses in these nanostructures. The $J_{\mathrm{sc}}$ of PSCs employing the Ag spheres, cylinders, and cubes increased by $8.9 \%, 9 \%$, and $3.5 \%$, respectively. The enhancement of the $\mathrm{TiO}_{2}$ nanostructured coatings was larger than that of their $\mathrm{Ag}$ counterparts because $\mathrm{TiO}_{2}$ had a smaller absorption cross-section and a larger 
scattering cross-section over the solar spectrum. The $J_{\mathrm{sc}}$ of PSCs with $\mathrm{TiO}_{2}$ spheres and cylinders increased by $12.8 \%$ and $15 \%$, respectively. Researchers also found that the metasurface cross-grating nanostructure within PSCs would increase the photocurrent and enhance the overall efficiency ${ }^{95}$. Enhanced light absorption and reduced light reflection are highly dependent on the dimensions, periodicity, and coating material of the grating nanostructures. $\mathrm{TiO}_{2}$ metasurfaces above and below the perovskite layer led to an increase in $J_{\mathrm{sc}}$ from 19.2 to 22.1 $\mathrm{mA} / \mathrm{cm}^{2}$, while the Au metasurfaces achieved a $J_{\mathrm{sc}}$ of $21.13 \mathrm{~mA} / \mathrm{cm}^{2}$.

More recently, Perrakis et al. presented detailed and systematic work on the implementation of metal NPs in $\mathrm{PSCs}^{96}$. CST Microwave Studio was used to perform 3D full-wave electromagnetic simulations. The PSC structure was $\mathrm{SiO}_{2}(1.1 \mathrm{~mm}) / \mathrm{ITO}(100 \mathrm{~nm}) /$ PEDOT:PSS $(40 \mathrm{~nm}) /$ $\mathrm{MAPbI}_{3} / \mathrm{PCBM}(50 \mathrm{~nm}) / \mathrm{Al}(100 \mathrm{~nm})$; the thickness of each layer is indicated in the brackets. Metal NPs with different materials, vertical positions, sizes, concentrations, clustering formations, and coatings were studied. It was demonstrated that (a) the optimum response of $\mathrm{Ag}$ NPs was with a radius of $60-70 \mathrm{~nm}$, especially when in the proximity of the top of the perovskite layer. Furthermore, the optimum NP distance was $\sim 300 \mathrm{~nm}$; (b) the effect of NP randomness, angle of incidence, and polydispersity was very limited on the calculated enhancement; (c) the $\mathrm{Al}$ nanospheres with radii of $18 \mathrm{~nm}$ and a gap distance of $65 \mathrm{~nm}$ had the optimum performance; (d) the photocurrent was largely enhanced (up to $4.0 \%$ corresponding to photocurrent density $\left(J_{\mathrm{ph}}\right)=21.22 \mathrm{~mA} / \mathrm{cm}^{2}$ ) when NPs were in the HTL (PEDOT:PSS, on top of the perovskite layer)and (e) the largest absorption enhancement was achieved when $\mathrm{Al}$ and $\mathrm{Ag}$ spheres were both used in the PEDOT:PSS layer and perovskite layer, respectively. Devices with Ag spheres embedded in the perovskite and $\mathrm{Al}$ spheres inside the HTL (PEDOT:PSS) can increase the PCE to $\sim 12 \%$. The origin of the photocurrent enhancement was attributed to the strong local $E$-field arising from the plasmonic resonances, and the larger scattering and antireflection due to the NPs, especially when the NPs were close to the top of the perovskite layer.

The above theoretical works studying the effect of plasmonic NPs on PSCs focused on characterizing PV performance, explaining the operational mechanism, and optimizing the NPs. Valuable primary results can be extracted from these theoretical models/simulations: (1) PV performance can be enhanced by incorporating NPs in PSCs; (2) the enhancement is due to the near-field absorption and far-field scattering of NPs induced by LSPR; (3) the geometry, size, material, density, distance, position, number, and coating all play important roles in enhancing PV performance. In addition, the absorption enhancement allows employment of PSCs with very thin perovskite layers, e.g., $150 \mathrm{~nm}$, while at the same time maintaining the same performance as that of nonplasmonic PSCs with the optimum thickness $(350 \mathrm{~nm})^{96}$. Therefore, plasmonic NPs significantly reduce toxicity and make PSCs more environmentally friendly. The simulation demonstrates that embedding plasmonic NPs into PSCs is a powerful strategy to improve photoelectric performance. Although many simulations have been performed, challenges and issues still exist. The simulations should involve more materials and geometries of NPs. Most of the simulations are focused on NPs embedded in perovskite, while more works studying the effects of NPs in the ETLs, HTLs, and combined layers are needed to provide more design possibilities. There are nonnegligible differences between the simulation models and realistic devices, such as deviations in the optical constants of the materials and the film quality in the real device not being as perfect as that in the simulations. Most of the simulations are focused on optical absorption for evaluating PV performance. Furthermore, the electrical properties of the metal, which lead to an increase in conductivity and electron-hole recombination, are not taken into account. This will bring about large discrepancies between the simulations and real devices. Overall, the simulations provide important theoretical guidelines, but the experimental ways of implementing NPs in PSCs still need to be explored.

\section{Experiments of NP-assisted perovskite solar cells}

Generally, plasmonic PSC experiments are focused on the configuration (NP position), modification of the NPs (material, geometry, size, and concentration), PV performance (PCE) enhancement, and underlying mechanism. The results are summarized in Table 1 in chronological order. Statistical analysis is performed based on these results with the following conclusions.

The position of the NPs can be in the ETLs (PCBM, $\mathrm{TiO}_{2}, \mathrm{Al}_{2} \mathrm{O}_{3}$, and $\mathrm{SnO}_{2}$ ), perovskite, and HTLs (spiroOMeTAD, PEDOT:PSS, and $\mathrm{VO}_{x}$ ). In simulations, perovskite is the major layer where the effect of NPs is studied, whereas the experiments mainly examine the use of plasmonic NPs in the ETLs and HTLs and at the ETL/HTL-perovskite interfaces. The NPs can be well blended into solutions of spiro-OMeTAD, PEDOT:PSS, and PCBM, which are then made into thin films as the building blocks of the device by facile spin-coating. Lee et al. found that when the spiro-OMeTAD HTL solution with Au NPs was spin-coated, the Au NPs could naturally stay close to the perovskite layer ${ }^{77}$. This rule likely also applies to the spin-coating of PEDOT:PSS and PCBM solutions with NPs. This distributes the NPs at the interface between the perovskite layer and spiroOMeTAD or PBCM layer because these two materials are typically spin-coated after the perovskite layer. 


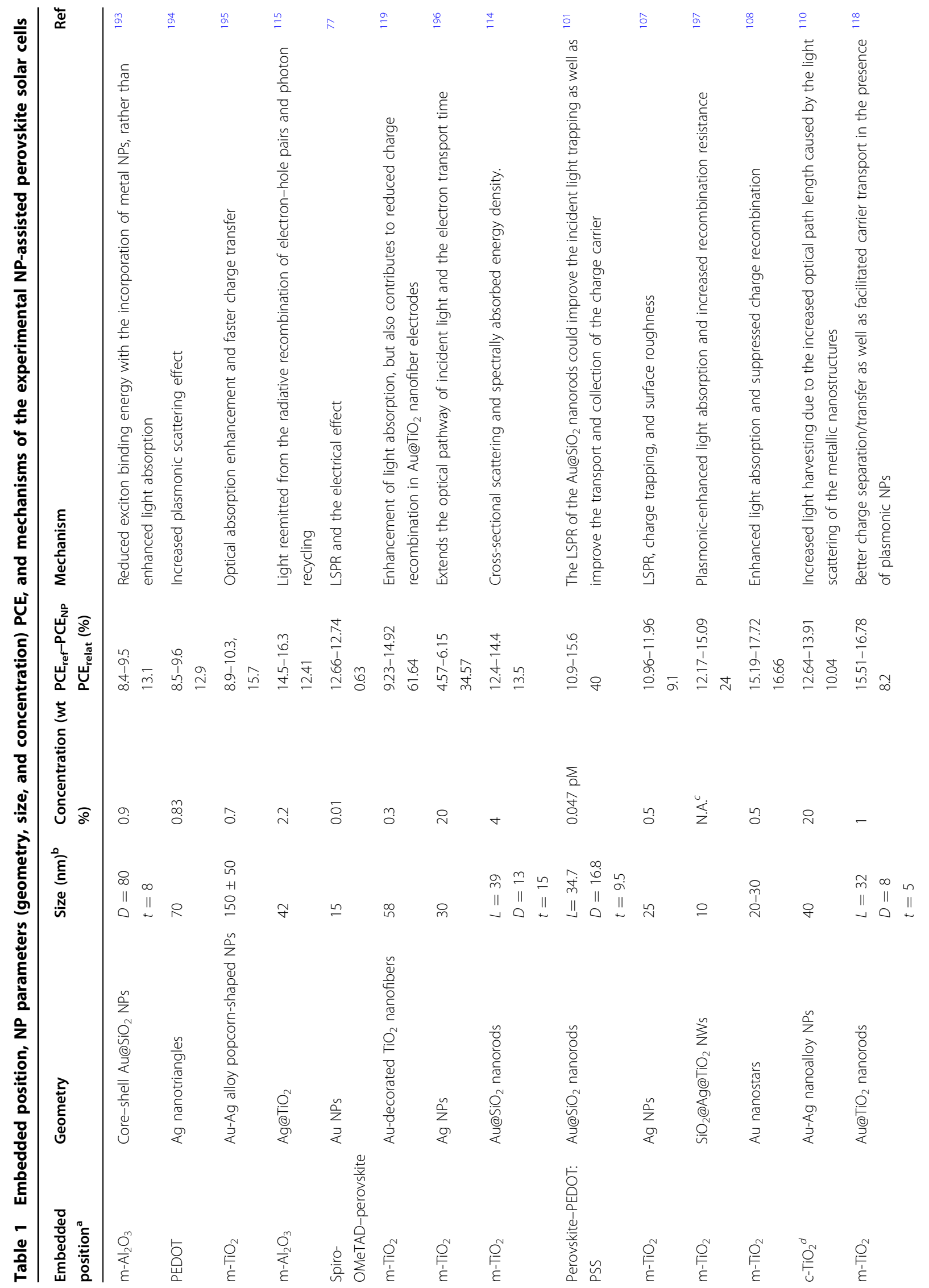




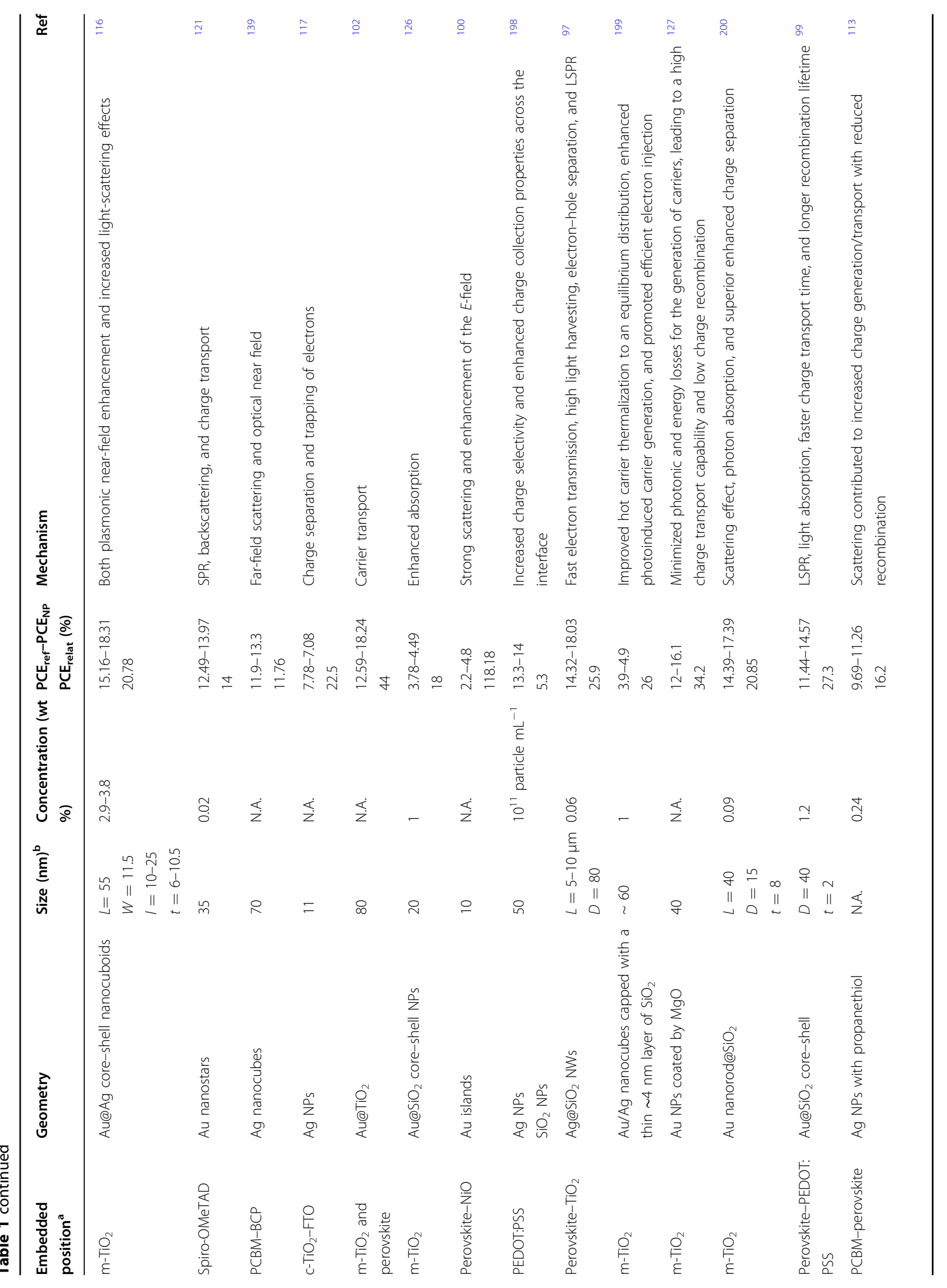




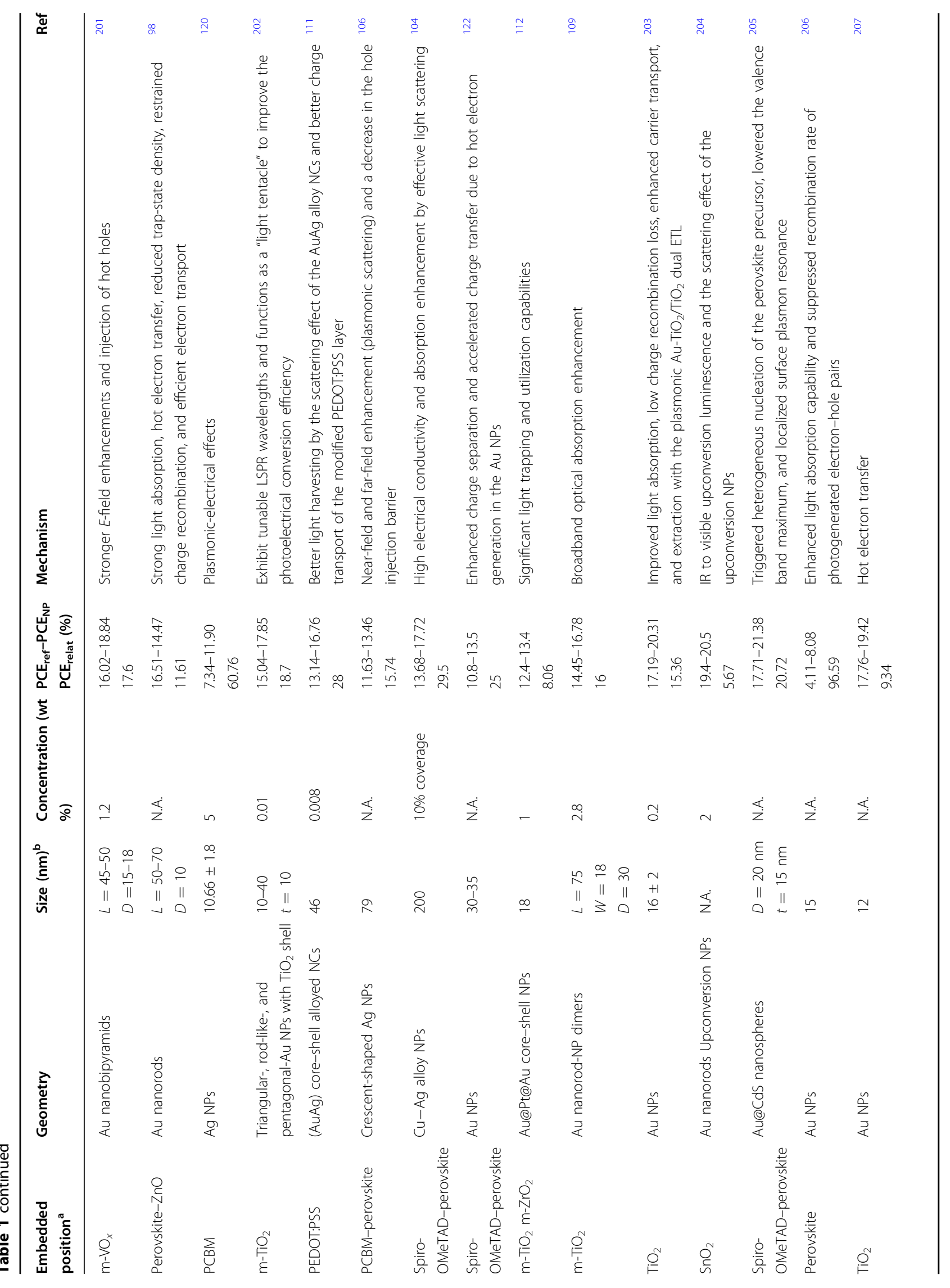




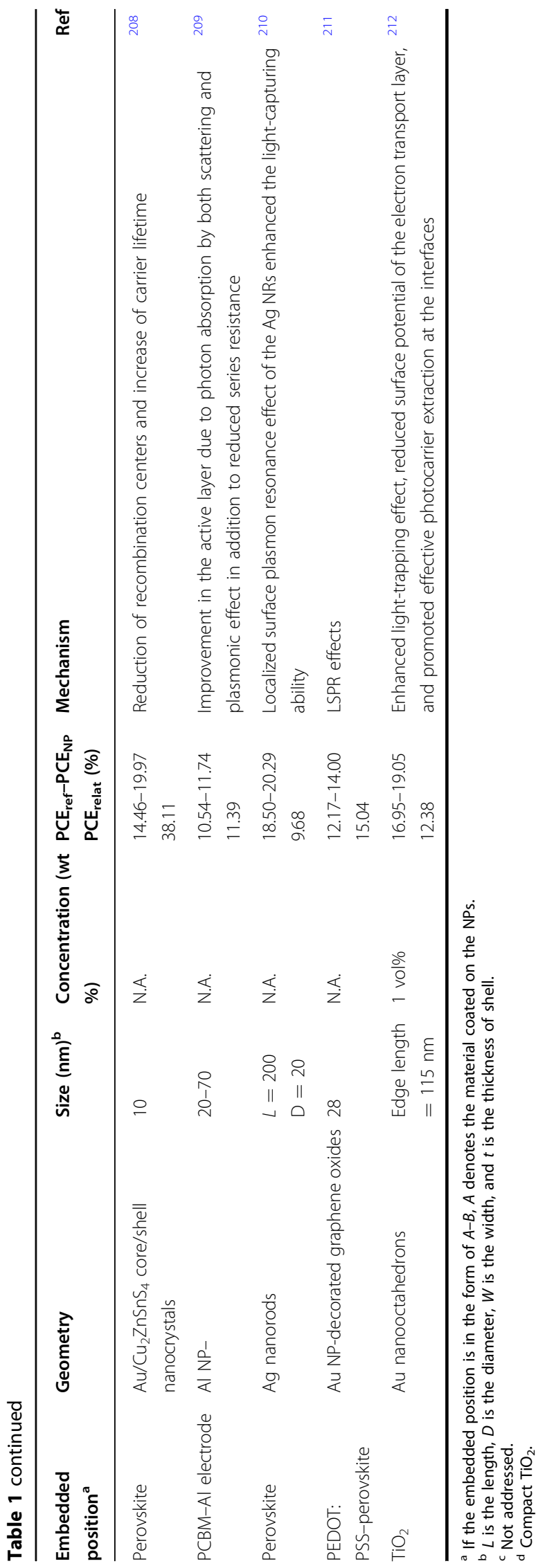

In contrast, the NPs prefer to stay far away from the perovskite in PEDOT:PSS, whose film formation is before the perovskite. The self-distribution of NPs by the spincoating method deserves a more detailed investigation, as it can be a good way to control the NP position. The spincoating of mixed solutions with NPs is also the major method to prepare NP-incorporated $\mathrm{TiO}_{2}, \mathrm{Al}_{2} \mathrm{O}_{3}$, and $\mathrm{VO}_{x}$ layers. They are mesoporous structures with a homogeneous distribution of NPs. NPs can also be incorporated in perovskites by spin-coating perovskite solutions blended with $\mathrm{NPs}^{97}$. However, in most cases, NPs are first deposited by spin-coating ${ }^{98}$, spraying ${ }^{99}$, or sputtering ${ }^{100}$, which are then coated by perovskite via spin-coating. Perovskite films with underlying NPs not only maintain their good quality but also show decreased roughness on the top perovskite surface and the absence of narrow gaps compared with perovskites without $\mathrm{NPs}^{99,101}$. It should be noted that one side of the NPs is attached on the surface of the $\mathrm{TiO}_{2}, \mathrm{Al}_{2} \mathrm{O}_{3}$, and $\mathrm{VO}_{x}$ scaffolds, while the other side is actually embedded in the perovskite, i.e., the NPs are at the perovskite-ETL/HTL interface. Analogously, for most other ETLs and HTLs, the NPs are at the perovskite-ETL/HTL interface. The position of the NPs is described by the form of the $A-B$ interface in the first column in Table 1, where A denotes the material coated on the NPs.

Figure 3a shows the ratio of the materials incorporated with NPs. The most commonly used material is $\mathrm{TiO}_{2}(22$ in 48 , i.e., $46 \%$ of the works), especially mesoporous $\mathrm{TiO}_{2}\left(\mathrm{~m}-\mathrm{TiO}_{2}, 17\right.$ works), followed by perovskites, spiroOMeTAD, PCBM, PEDOT:PSS, $\mathrm{Al}_{2} \mathrm{O}_{3}, \mathrm{VO}_{x}$, and $\mathrm{SnO}_{2}$. The PCEs of the reference devices without NPs and the devices with NPs are denoted as $\mathrm{PCE}_{\mathrm{ref}}$ and $\mathrm{PCE}_{\mathrm{NP}}$, respectively. The relative enhancement is calculated as $\mathrm{PCE}_{\text {relat }}=\frac{\mathrm{PCE}_{\mathrm{NP}}-\mathrm{PCE}_{\text {ref }}}{\mathrm{PCE}_{\text {ref }}} \times 100 \%$. The $\mathrm{PCE}_{\mathrm{ref}}, \mathrm{PCE}_{\mathrm{NP}}$, and $\mathrm{PCE}_{\text {relat }}$ are listed in Table 1. For a fair comparison of the different incorporated materials, we exclude devices with $\mathrm{PCE}_{\text {ref }}<10 \%$. For the rest of the devices, the $\mathrm{PCE}_{\text {relat }}$ is analyzed by the maximum (max) $\mathrm{PCE}_{\text {relat }}$ (using any NPs), average plain $\mathrm{PCE}_{\text {relat }}$ (only using simple NPs with a spherical geometry and materials of $\mathrm{Au}$ or $\mathrm{Ag}$ ), and the average $\mathrm{PCE}_{\text {relat }}$ (Fig. 3b). NPs incorporated in the perovskite layer show a maximum $\mathrm{PCE}_{\text {relat }}$ of $40 \%$, while NPs incorporated in the $\mathrm{TiO}_{2}$ layer are slightly lower (34.2\%). NPs incorporated in other material layers achieve lower efficiencies. Simple NPs are mainly incorporated in spiroOMeTAD, PEDOT:PSS, and $\mathrm{TiO}_{2}$. The plain $\mathrm{PCE}_{\text {relat }}$ of spiro-OMeTAD is the highest, but in general, the plain $\mathrm{PCE}_{\text {relat }}$ is lower than the average $\mathrm{PCE}_{\text {relat }}$. The highest average $\mathrm{PCE}_{\text {relat }}(25.4 \%)$ is found in PSCs where NPs are located within the perovskite layer. This is followed by NPs embedded in spiro-OMeTAD, $\mathrm{VO}_{x}, \mathrm{TiO}_{2}$ and PEDOT:PSS, with an average PCE $_{\text {relat }}$ between $16 \%$ and $18 \%$. In comparison, NPs incorporated in PCBM and 


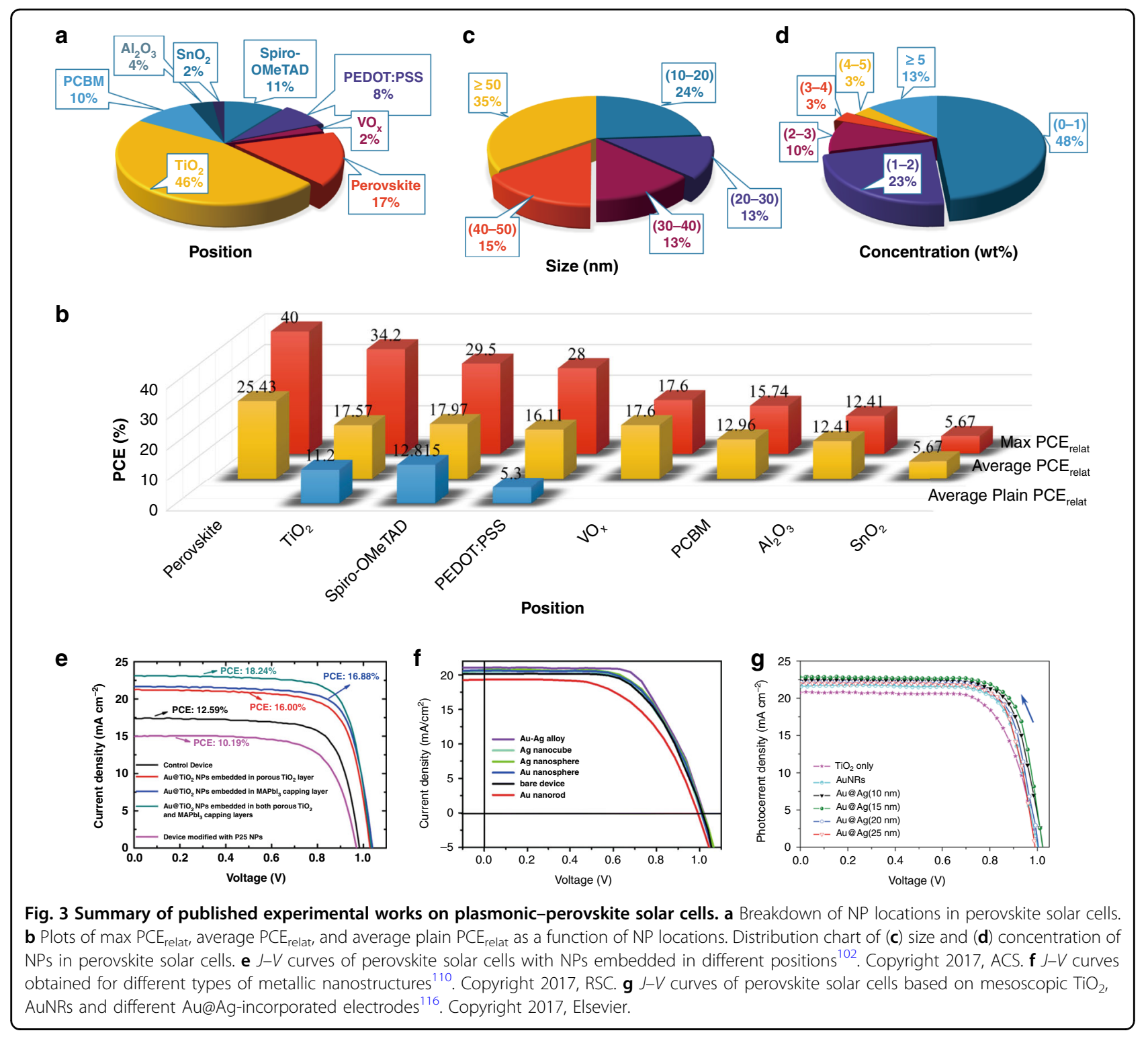

$\mathrm{Al}_{2} \mathrm{O}_{3}$ produce an inferior average $\mathrm{PCE}_{\text {relat }}$ in the $12 \%$ to $13 \%$ range, while NPs incorporated in $\mathrm{SnO}_{2}$ deliver the lowest average $\mathrm{PCE}_{\text {relat }}$ (below 6\%). Overall, perovskite and $\mathrm{TiO}_{2}$ seem to be the best material layers for incorporating NPs to achieve a large PCE enhancement, especially when NPs are positioned close to the perovskite- $\mathrm{m}-\mathrm{TiO}_{2}$ interface. Interestingly, Luo et al. incorporated $\mathrm{Au} @ \mathrm{TiO}_{2} \mathrm{NPs}$ into both $\mathrm{m}-\mathrm{TiO}_{2}$ and perovskite ${ }^{102}$ and obtained a maximum $\mathrm{PCE}_{\text {relat }}$ of $44 \%$ (Fig. 3e), which is the largest enhancement to the best of our knowledge. This result suggests that incorporating NPs in more than one position may achieve a higher efficiency, thus encouraging further exploration of multipositioned plasmonic NPs in PSCs.

The geometry of NPs varies in the form of spheres, nanostars, core-shell NPs, NWs, crescents, nanorods, nanocubes, triangles, pentagons, nanofibers, islands, nanobipyramids, popcorns, and nanorod dimers. NPs are either synthesized first by the seed-mediated growth method before incorporation or directly prepared in building blocks by using block copolymer micelle lithography $(\mathrm{BCML})^{103}$, the wet chemical method ${ }^{104}$, the seedmediated method ${ }^{105}$, thermal deposition ${ }^{106}$, and sputtering $^{100}$. We classify the NPs into four types of structures: simple spherical NPs, core-shell NPs, shape-modified NPs, and dimers. Spherical Ag-NPs have been embedded into the $\mathrm{TiO}_{2}$ photoanode layer ${ }^{107}$, and the PCE of the PSC with Ag-NPs increased to $11.96 \%$ from 10.96\%, which was a $9.1 \%$ enhancement. $\mathrm{Li}$ et al. integrated $\mathrm{Au} @ \mathrm{TiO}_{2}$ core-shell (@ means $\mathrm{Au}$ is the core and $\mathrm{TiO}_{2}$ is the shell) NPs into $\mathrm{m}-\mathrm{TiO}_{2}$ and/or perovskite semiconductor capping layers ${ }^{102}$, enhancing the PCE from 
$12.59 \%$ (reference device without metal NPs) to $18.24 \%$ (Fig. 3e). Plasmonic Au nanostars have been incorporated into $\mathrm{m}-\mathrm{TiO}_{2}$ for $\mathrm{PSCs}^{108}$, and the PSCs fabricated with $\mathrm{TiO}_{2}-\mathrm{Au}$ nanostars exhibited a PCE enhancement of $16.66 \%$, increasing from $15.19 \%$ to $17.72 \%$. Ma et al. showed that Au nanorod-NP dimers could enhance the PCE of PSCs by $16 \%{ }^{109}$. The NPs of simple spherical NPs, core-shell NPs, shape-modified NPs, and dimers all brought about remarkable PCE enhancements. In particular, the latter three structures brought about higher PCEs than simple spherical NPs.

Materials such as $\mathrm{Ag}, \mathrm{Au}, \mathrm{Au} @ \mathrm{SiO}_{2}, \mathrm{Ag} @ \mathrm{TiO}_{2}$, $\mathrm{SiO}_{2} @ \mathrm{Ag} @ \mathrm{TiO}_{2}, \mathrm{Au} @ \mathrm{Ag}, \mathrm{Au}-\mathrm{Ag}$ alloy, Au@Pt@Au, and $\mathrm{Cu}-\mathrm{Ag}$ alloy were used to fabricate NPs. For instance, $\mathrm{Au}$, $\mathrm{Ag}$, and $\mathrm{Au}-\mathrm{Ag}$ nanoalloy NPs with different sizes and shapes were embedded into the ETLs $\left(\mathrm{TiO}_{2}\right)$ of PSCs via the physical deposition method ${ }^{110}$. The $\mathrm{Au}-\mathrm{Ag}$ nanoalloy showed the best PCE improvement with a PCE $=14.8 \%$, which was a PCE increase of $17.5 \%$ compared with bare PSCs (Fig. 3f). Sun et al. applied AuAg@AuAg (AuAg) core-shell alloy NCs (ANCs) into the PEDOT:PSS layers of PSCs as a dopant ${ }^{111}$. The highest PCE of the devices with AuAg ANC-doped PEDOT:PSS was 16.76\%, which was an increase of $28 \%$ compared with that of the devices without AuAg ANCs (PCE = 13.14\%). Chen et al. incorporated $\mathrm{Cu}-\mathrm{Ag}$ alloy NPs to achieve a remarkable efficiency of $18.89 \%{ }^{104}$. Au@Pt@Au core-shell NPs were incorporated into PSCs ${ }^{112}$, which increased the PCE by 8.1\%, from $12.4 \%$ (normal PSCs without NPs) to $13.4 \%$. It should be noted that NPs are usually coated by ligands (polyvinylpyrrolidone (PVP)) after the solution synthesis process ${ }^{107}$. Cho et al. replaced the original capping agent (PVP) with thermally stable propanethiol ligands by a ligand exchange method, which can prevent the aggregation of Ag NPs in the perovskite active layer ${ }^{113}$. The propanethiol ligands can improve the optical and morphological properties and lead to a better dispersion of the Ag NPs in the perovskite than that of the PVP-capped or bare Ag NPs. Regarding the core-shell structure, the shells can be $\mathrm{SiO}_{2}{ }^{114}$ and $\mathrm{TiO}_{2}{ }^{115}$. Tang et al. demonstrated that $\mathrm{Au} @ \mathrm{SiO}_{2}$ core-shell nanorods with optimized aspect ratios could greatly enhance the performance of $\mathrm{MAPbI}_{3} \mathrm{PSCs}^{114}$. Such structures achieved a $16.1 \%$ improvement (from $12.4 \%$ to $14.4 \%$ ) in the maximal EQE and increased the maximal $J_{\mathrm{sc}}$ by $13.5 \%$ from 20.0 to $22.7 \mathrm{~mA} / \mathrm{cm}^{2}$. The open voltage $\left(V_{\text {oc }}\right)$ and filling factor (FF) remained almost the same. Core-shell

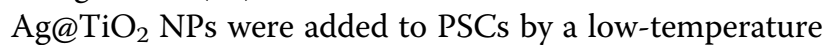
processing route, enhancing the PCE up to $16.3 \%{ }^{115}$. In general, the alloyed NPs show stronger enhancement.

Figure 3c, d shows the distribution of the sizes and concentrations of NPs that possess the largest PCE enhancement in each work. Approximately $90 \%$ of the works fall in the size range of $10-50 \mathrm{~nm}$ and concentration range of $0-5 \mathrm{wt} \%$. Approximately half of the works use small NPs $(<30 \mathrm{~nm})$ and low concentrations $(<1 \mathrm{wt} \%)$. Detailed results are presented to study the size effect. Au@Ag nanocuboids with lengths of 10, 15, 20, and $25 \mathrm{~nm}$ have been incorporated into an $\mathrm{m}-\mathrm{TiO}_{2}$ layer, yielding PCEs of $16.89 \%, 17.21 \%, 16.35 \%$, and $16.14 \%$, respectively; these values correspond to relative enhancements of $11.4 \%, 13.5 \%, 7.8 \%$, and $6.5 \%$ (Fig. 3g), respectively ${ }^{116}$. Au@Ag nanocuboids with a diameter of $15 \mathrm{~nm}$ show the best performance. Liu et al. investigated the effect of Ag NPs with diameters of 11 and $16 \mathrm{~nm}$ on the performance of PSCs ${ }^{117}$. PSCs with different NPs showed different $J_{\mathrm{sc}}, V_{\mathrm{oc}}$, and $F F$ values. Overall, the NPs with a diameter of $11 \mathrm{~nm}$ showed the largest PCE. Regarding core-shell NPs, the thickness of the shell is important. Pathak et al. investigated the effect of the shell thickness by coating $\mathrm{Au}$ NRs with 5 and $10 \mathrm{~nm} \mathrm{TiO}_{2}$ shells ${ }^{118}$. The performance of all devices with the $\mathrm{Au} @ \mathrm{TiO}_{2}$ nanorods (NRs) improved, regardless of the $\mathrm{TiO}_{2}$ shell thickness. However, their underlying mechanism for the performance enhancement was different. The $J_{\text {sc }}$ of the devices with the $\mathrm{Au} @ \mathrm{TiO}_{2} \mathrm{NRs}$ that had thicker shells remained the same, but their $V_{\text {oc }}$ increased from 1.02 to $1.08 \mathrm{~V}$ compared with the devices without the NRs. In contrast, regarding the devices with thinner shells, $V_{\mathrm{oc}}$ remained unchanged, while $J_{\mathrm{sc}}$ exhibited an improvement from 20.68 to $22.23 \mathrm{~mA} / \mathrm{cm}^{2}$. Particular efforts have also been devoted to the concentration effect. Malireport et al. studied the effect of $0.1 \mathrm{wt} \%$ to 0.5 wt\% $\mathrm{Au}$ NPs in $\mathrm{TiO}_{2}$ on PSCs based on an in situ synthesis method of Au-embedded $\mathrm{TiO}_{2}$ nanofibers by an electrospinning technique ${ }^{119}$. The PCE, $V_{\mathrm{oc}}, J_{\mathrm{sc}}$, and $F F$ increased as the NP concentration was increased from 0.1 wt $\%$ to $0.3 \mathrm{wt} \%$; notably, these parameters decreased when the NP concentration was increased from $0.3 \mathrm{wt} \%$ to 0.5 $w t \%$, i.e., the devices with $0.3 \mathrm{wt} \%$ attained the highest performance. Higgins et al. demonstrated planar PSCs with the structure ITO/PEDOT:PSS/MAPbI $/ \mathrm{PCBM}_{3} / \mathrm{Ni}$ : $\mathrm{Au}$, where PCBM was modified with variable amounts of Ag NPs $(3,5,7,10,20 \text {, and } 100 \mathrm{wt} \%)^{120}$. The addition of 5 wt\% Ag NPs showed the largest PCE enhancement of $60.76 \%$, increasing from $7.34 \%$ to $11.90 \%$. Based on the above analysis, the size and concentration have different effects on the current, voltage, and $F F$ and thus need to be optimized carefully to achieve the best performance.

The underlying mechanism of plasmonic NPs in PSCs was discussed in previous reports. The keywords are presented in Table 1, including the LSPR, field enhancement, electron-hole separation, scattering, charge separation, light absorption, hot electron injection, and carrier transport. At times, the explanations are confusing and even conflicting. Here, we attempt to provide a comprehensive and consistent summary of the mechanisms by looking into the cause and effect, as shown in 
a

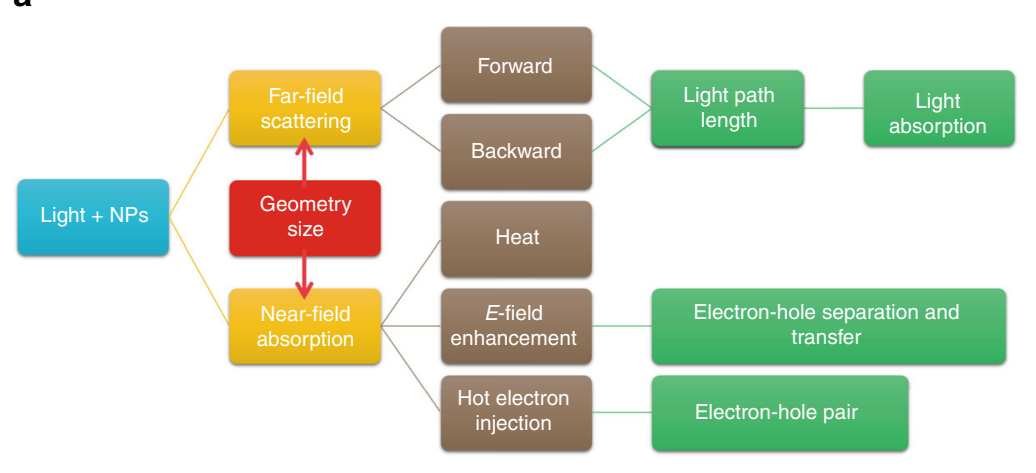

b

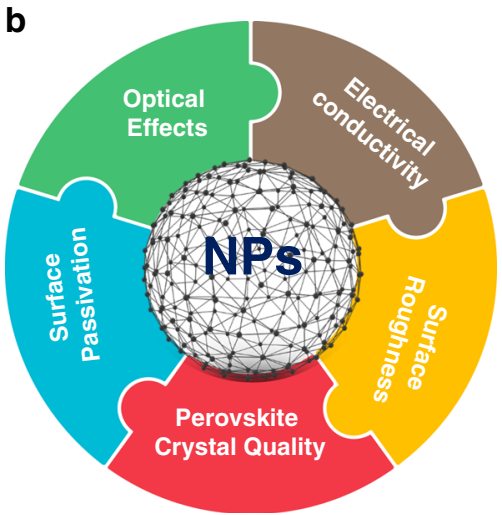

Fig. 4 Mechanisms behind NP-assisted perovskite solar cells. a Far-field scattering and near-field absorption can both occur upon light illumination of NPs. Forward and backward far-field scattering will increase the optical path length, which leads to more absorption events within the light-absorbing semiconductor. Near-field absorption enhances the electric field, photothermal conversion, and hot electron injection. E-field enhancement brings stronger oscillation energy for faster electron-hole separation and electron (hole) transfer. Electrons will escape from the metal NPs to the active layer, inducing hot electron injection. This phenomenon provides extra electron-hole pairs for current extraction. $\mathbf{b}$ In addition to the optical effects, the incorporation of plasmonic nanoparticles in perovskite materials also influences the electrical conductivity, surface roughness, perovskite crystal quality, and surface passivation, which are all crucial factors to realize highly efficient and stable perovskite solar cells.

Fig. 4a. When light illuminates NPs, optical effects of near-field absorption and far-field scattering are generated. When the NPs are in front of the perovskite, i.e., light first reaches the NPs, which occurs for the PEDOT: PSS and $\mathrm{TiO}_{2}$ layers, forward scattering will increase the optical path length and enhance the light absorption of the perovskite, while backward scattering will produce a negative effect. Backward scattering will be beneficial if the NPs are placed beneath the perovskite (at the rear side closest to the metal electrode), for example, in the spiro$\mathrm{OMeTAD}^{121}$ and PCBM ${ }^{106}$ layers. $J_{\text {sc }}$ will improve due to enhanced light harvesting. The scattering effect depends on the size of the NPs. Large particles $(>100 \mathrm{~nm})$ are deemed more appropriate for scattering in PV applications $^{70}$. However, the sizes of the NPs in $90 \%$ of the previous works are smaller than $50 \mathrm{~nm}$ (Fig. 3c). This indicates that the scattering effect of the NPs in the PSCs is minor. Near-field absorption leads to effects such as heat generation, $E$-field enhancement, and hot electron injection. As the light intensity is low, photothermal heating may be limited, which itself is interesting to verify experimentally. When NPs are in the perovskite, especially at the perovskite interface, the $E$-field enhancement brings about stronger oscillation energy for faster electron-hole separation and electron (hole) transfer, i.e., higher recombination resistance, resulting in a larger $F F$. Hot electron injection provides extra electron-hole pairs and thus leads to an improved $J_{\mathrm{sc}}{ }^{118,122}$. However, if the NPs are incorporated in the ETLs/HTLs and far away from the perovskite, the contribution of the near-field absorption effects is much reduced or even becomes negative. In addition to the light-NP interaction, other effects also take place (Fig. 4b). Metal NPs with dielectric shells improve the electrical conductivity, decrease the device resistance, and increase the $J_{\mathrm{sc}}$. In contrast, bare NPs and the agglomeration of NPs create charge recombination centers, which in turn degrade the overall PV performance. The surface roughness at the interfacial area between the carrier transport layer and perovskite layer increases due to the incorporation of NPs. The rough surface leads to a larger surface area and thus allows the collection of a larger number of photogenerated carriers $^{123}$. The perovskite coverage can be increased by spin-coating PCBM with NPs because NPs can fill the empty spaces and passivate the grain boundaries ${ }^{120}$. NPs can also act as nucleation sites for perovskite films to improve the crystal quality ${ }^{124,125}$.

In summary, the PV performance of PSCs can be enhanced by plasmonic NPs because NPs lead to overall positive effects. The following conclusions are extracted based on the analysis of previous works: (1) The best position for using plasmonic NPs in PSCs is the perovskite- $\mathrm{m}-\mathrm{TiO}_{2}$ interface because $\mathrm{m}-\mathrm{TiO}_{2}$ is one of the best ETL materials that has good conductivity and a large surface area, and the plasmonic effects can both enhance the light-matter interaction of the perovskite and $\mathrm{m}-\mathrm{TiO}_{2}$ layers. (2) NPs with core-shell structures, modified shapes, and dimers show stronger effects than simple spherical NPs. Bare NPs without any coating reduce PV performance $\left(V_{\mathrm{oc}}\right)$ because they act as carrier recombination centers ${ }^{117}$. Hence, NPs are usually coated by ligands ${ }^{113}$, dielectric shells $\left(\mathrm{SiO}_{2} \text { and } \mathrm{TiO}_{2}\right)^{126}$, or additional dielectric layers ${ }^{127}$. Two resonance modes are excited along the long and short axes of the nanorods. This enhances the near-field absorption over a broader frequency bandwidth. The tips of NPs, such as triangles, 
cubes, pentagons, and popcorns, provide more hotspots than spherical NPs, leading to stronger LSPR and nearfield absorption. NP dimers with small gaps have enhanced near-field absorption due to the stronger coupling between NPs. NPs with core-shell, dimer, and other modified shapes also show stronger effects than simple spherical NPs. (3) The alloyed NPs show a larger PV enhancement than that of a single material because the composite materials can broaden the absorption spectrum. (4) The size and concentration have different effects on the current, voltage, and $F F$ and thus need to be optimized carefully. The size of the NPs should be tuned to make the absorption of NPs fall within the solar spectrum and overlap as much as possible, especially in the wavelength range where the intrinsic absorption of the perovskite is weak. Ultraviolet (UV) and NIR light can be absorbed by controlling the size of the NPs. In addition, the thickness of the shell is important. The interaction between the near-field absorption and perovskite can be changed due to the different shell thicknesses. When the shell is thick, the surface potential of the coating dielectric layer is changed by hot electron injection. As a result, the built-in potential of the device will increase ${ }^{118}$. The $V_{\mathrm{oc}}$ enhancement is not obvious once the shell is thinner than a certain threshold. In contrast, $J_{\mathrm{sc}}$ will be improved with a thinner dielectric layer because the interaction between the hot electrons from the metal and the excitons from the perovskite is strong and facilitates carrier separation and transport within the devices. A larger concentration of NPs results in stronger absorption; however, this also leads to agglomeration and recombination centers ${ }^{119}$. The majority of the NPs achieve a maximum PCE at a low concentration of $<2 \mathrm{wt} \%$ (Fig. 3d). Based on the summarized results, we infer that alloyed core-shell nanorods (with sharp tips and sub-10 $\mathrm{nm}$ gaps) that are dispersed at the perovskite-m- $\mathrm{TiO}_{2}$ interface show promise for achieving higher PV performance. The sizes and concentrations may be optimal when $<50 \mathrm{~nm}$ and $<2 \mathrm{wt} \%$, respectively.

\section{Plasmonic film-assisted perovskite solar cells}

Plasmonic films are defined here as corrugated structures such as periodic arrays or random distributions of subwavelength holes (nanoholes) perforated in a metal film ${ }^{128,129}$ and structured continuous films ${ }^{130,131}$ that can excite the SPP mode or both the SPP and LSPR modes ${ }^{132}$. The plasmonic properties can be tuned by the film morphology, periodicity, and materials. The coupling between SPPs and LSPRs can result in unique optical effects and stronger light confinement. In addition, the penetration depth of SPPs into the dielectric can be $100 \mathrm{~nm}-1 \mu \mathrm{m}$, which is much larger than that of LSPR $(<10 \mathrm{~nm})^{133}$. This makes SPPs, in principle, able to exert a larger influence on the perovskite layer, ETL or HTL, leading to a higher PCE.
Numerous simulations have been carried out to provide theoretical guidelines. Nanohemispherical Au electrodes have been designed for PSCs (Fig. 5a) ${ }^{134,135}$. The numerical results based on the FEM indicate that light absorption in these 2D photonic-structured PSCs is apparently stronger than that in a planar PSC. The integrated absorption efficiency reaches $65.7 \%$ over wavelengths ranging from 350 to $800 \mathrm{~nm}$ at normal incidence considering an air mass (AM) of 1.5 G solar irradiation, with an enhancement of $88.3 \%$ of that of the planar PSC. This great enhancement in light absorption is attributed to the excitation of the diverse plasmonic and photonic modes, including dipole-like LSPR, SPP modes, Bloch modes, cavity modes, and their mutual couplings. Abdelraouf et al. proposed a theoretical PSC model with front dielectric and back plasmonic wire gratings (Fig. $5 \mathrm{~b})^{136}$. The cross-sections of the grating with equally spaced wires were rectangular, triangular, and hemispherical. The light absorption was the highest for $\mathrm{Ag}$ rectangular wire gratings with side lengths of 110-150 $\mathrm{nm}$, and $J_{\mathrm{sc}}$ increased by $22.4 \%$ compared with that of the planar structure. The $J_{\mathrm{sc}}$ of $\mathrm{Ag}$ triangular and semispherical wire gratings increased by $13.9 \%$ and $15.4 \%$, respectively. The enhancement arose from both the contribution of the front dielectric wire grating and back plasmonic wire grating, with the former controlling the light-scattering directivity through Mie resonance and the latter solving the problem of low light absorption at longer wavelengths.

In experiments, Long et al. fabricated a periodic corrugated HTL and Au electrode ${ }^{137}$ (Fig. 5c). The absorption was stronger for the periodic microstructure composite HTL with the conformal Au electrode compared with the planar HTL and electrode due to enhanced cavity effects. Both Fabry-Pérot resonance and SPR played a role in the cavity, increasing the light-harvesting efficiency through the coupling between light and surface plasmons. Wei et al. fabricated a metal back electrode into a moth-eye nanostructure via a soft imprinting technique (Fig. 5d,e $)^{138}$. Compared with the flat reference cell, the $J_{\mathrm{sc}}$ of the patterned devices with moth-eye nanostructures was enhanced by $14.3 \%$. Its PCE was up to $16.31 \%$ without sacrificing the $V_{\text {oc }}$ and FF. The moth-eye back electrode enhanced the light scattering over a broad frequency band and induced plasmonic effects for stronger light confinement in the device, leading to performance enhancements. The combination of metallic films and NPs has also been explored. Kim et al. developed planar PSCs with both plasmonic $\mathrm{Ag}$ nanocubes and $\mathrm{Ag}$ back electrode films ${ }^{139}$. An ETL was inserted between the nanocubes and electrode, whose thickness was varied to tune the coupling between the Ag nanocubes and the back electrode to make the plasmonic absorption peak close to the absorption edge of the perovskite layer. The far-field 


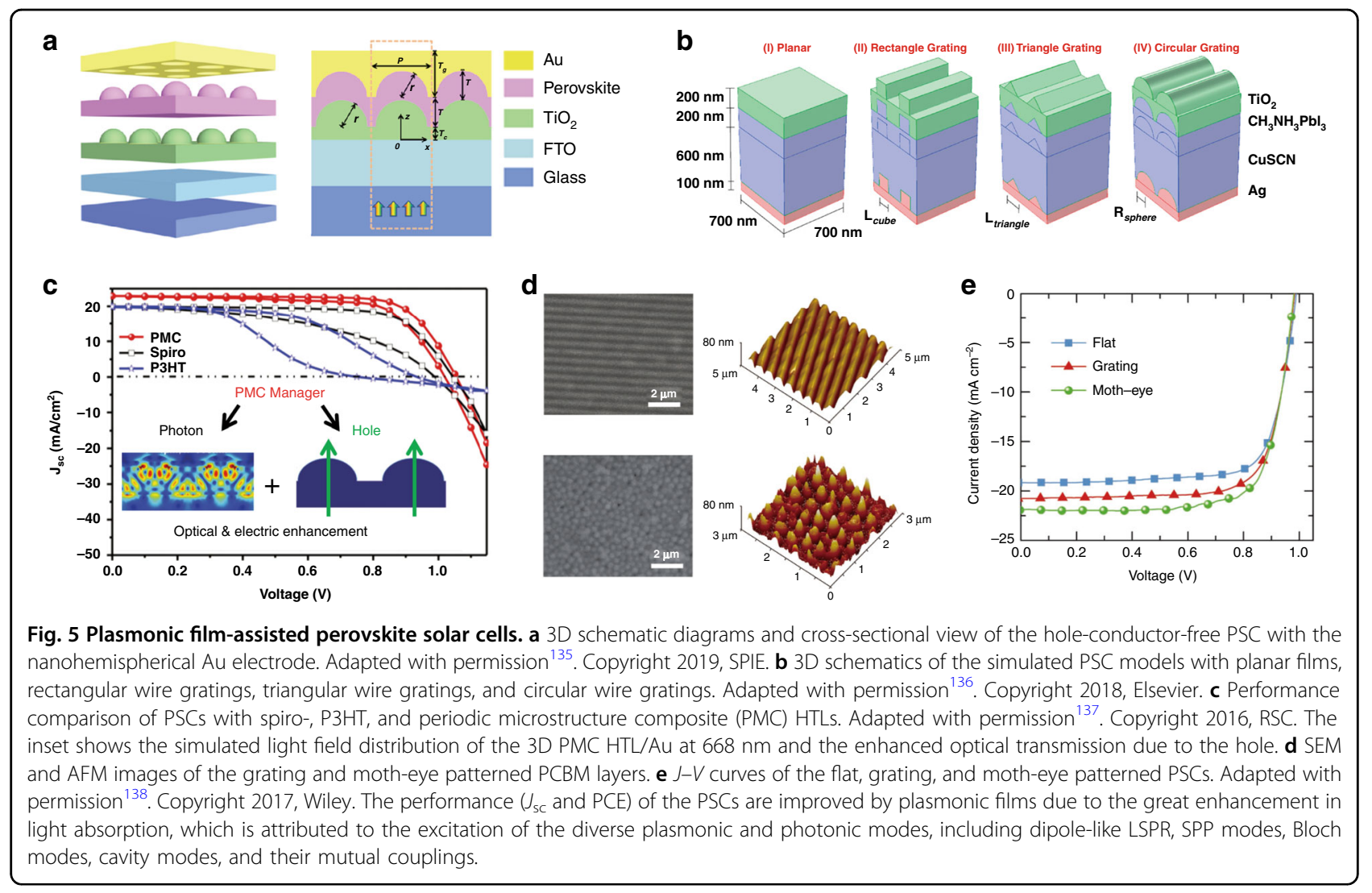

scattering and near-field absorption around the nanocube face closest to the perovskite layer were also greatly enhanced by the coupling. The average PCE was enhanced from $11.9 \%$ to $13.3 \%$ due to plasmonic coupling.

Plasmonic films have not been used as widely as NPs because the fabrication of corrugated plasmonic films in PSCs is usually more complex. In addition, the transmission of plasmonic films is low, so plasmonic films can only be used on the rear side of PSCs. Nevertheless, these pioneering works have shown the great potential of plasmonic films to increase the efficiency of PSCs.

\section{Plasmonic-perovskite light emitters \\ Enhanced spontaneous emission}

Halide perovskite materials exhibit strong spontaneous emission, whose wavelengths can be tuned throughout the visible range by mixing halide anions. This makes perovskites a promising platform for creating efficient, low-cost and multicolor optoelectronic devices such as LEDs and displays. Incorporating plasmonic nanostructures into LEDs to induce coupling between excitons (i.e., electron-hole pairs bound by Coulombic interactions) and surface plasmons is a powerful strategy to enhance the performance of perovskite LEDs (Fig. 6a) ${ }^{140}$. In theory, if the energy of the excitons of perovskite matches the SP resonant energy, the two energies will couple and enhance the scattering or free-space reemission. This leads to an extra recombination pathway, which significantly increases the spontaneous radiation rate (Fig. 6b) and improves emission performance.

The plasmonic effects on perovskite LEDs are studied mainly based on NPs. Mokkath et al. calculated the optical properties of Au-doped all-inorganic $\mathrm{CsPbX}_{3}(X=\mathrm{Cl}, \mathrm{Br}$, I) perovskite quantum dots (QDs) based on timedependent density functional theory ${ }^{141}$. Their results confirmed deep-level trap states induced by the $\mathrm{Au}$ dopant, a significant absorption redshift, and the emergence of $E$-field hotspot regions. $\mathrm{CsPBBr}_{3} @ \mathrm{Ag}$ hybrid NCs were synthesized by reacting $\mathrm{CsPBrr}_{3} \mathrm{NCs}$ with $\mathrm{Ag} X(X=$ $\mathrm{Cl}, \mathrm{Br}$, or I) powders in hexane ${ }^{142}$. Briefly, 2-5 nm Ag NPs were nucleated and attached randomly on the surface of $\mathrm{CsPbBr}_{3}$ NCs. The PL intensity and emission lifetime of the $\mathrm{CsPbBr}_{3} @ \mathrm{Ag}$ hybrid NCs were significantly enhanced and reduced, respectively, compared with those of pure $\mathrm{CsPbBr}_{3} \mathrm{NCs}$ when illuminated by $400 \mathrm{~nm}$ light (Fig. 6c, d). The enhancement was mainly attributed to the plasmonic effects of the Ag NPs enhancing the absorbance of UV or blue light. However, Ag adhesion deteriorated the surface quality of the $\mathrm{CsPBr}_{3} \mathrm{NCs}$ and in turn undermined the PLQY. Therefore, Ag adhesion has both positive and negative effects on the PL of hybrid perovskite QDs. To achieve an enhanced PL, the size and density of 

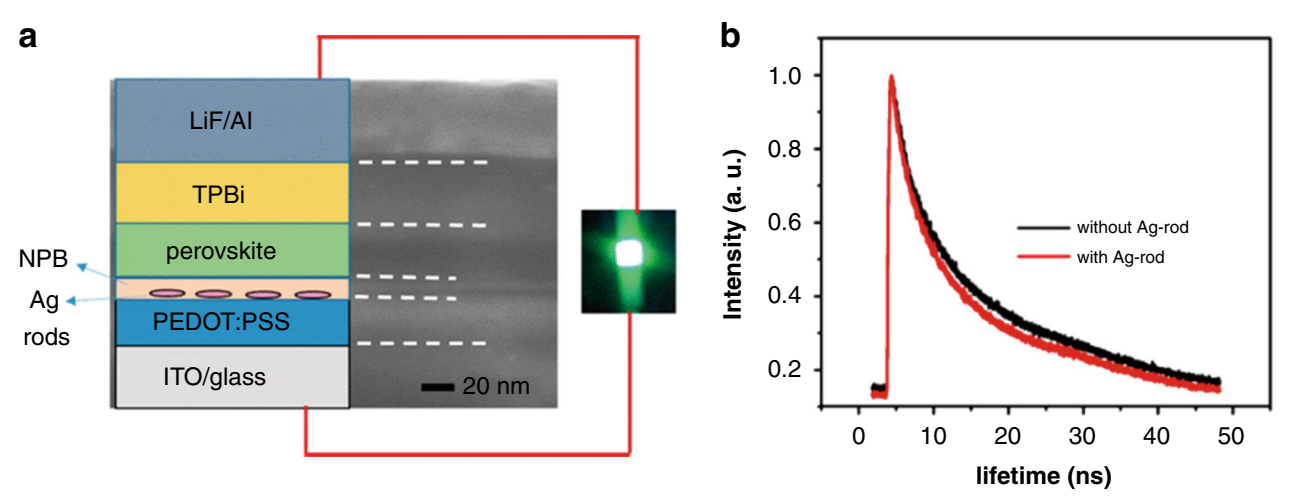

C

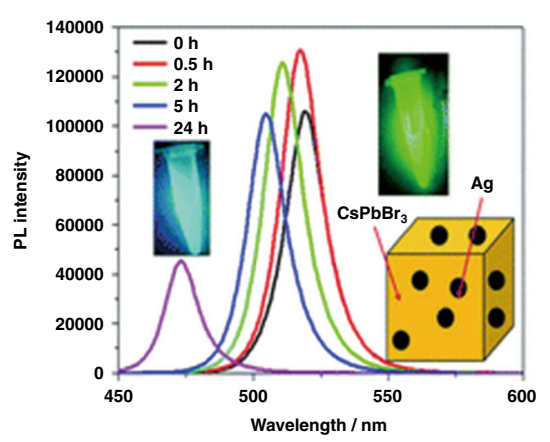

e

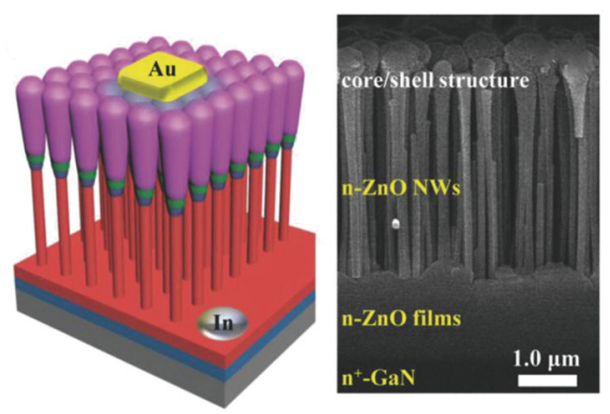

d
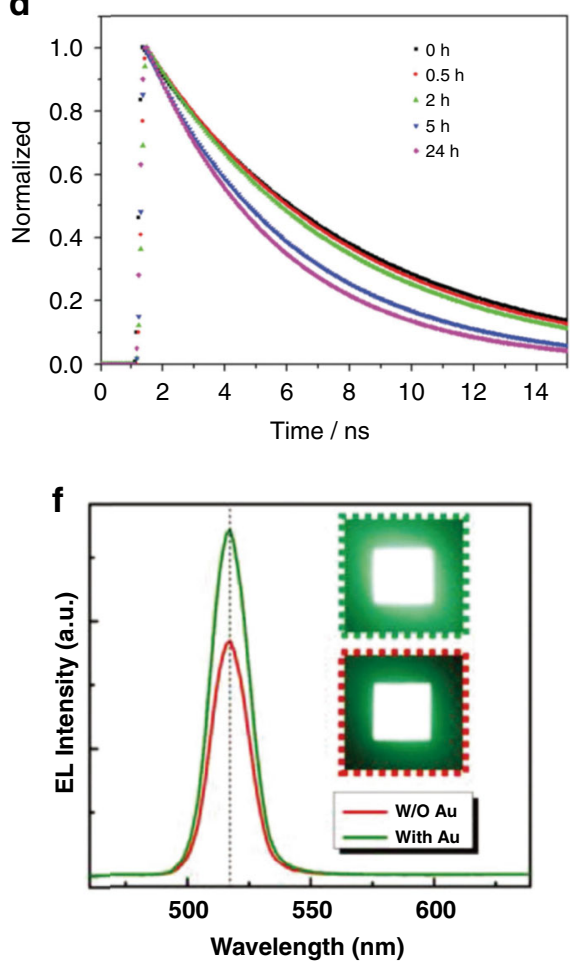

Fig. 6 Plasmonic-perovskite light emitters with enhanced spontaneous emission. a TEM image of a cross-section, the corresponding schematic diagram, and the photographic image of the plasmonic-perovskite LED based on the Ag-CsPbBr 3 system. Adapted with permission ${ }^{140}$. Copyright 2017, ACS. b PL lifetime of the perovskite $\mathrm{CsPbBr}_{3}$ nanocrystal layer with and without Ag rods. c PL intensity and (d) fitted time-resolved photoluminescence decay spectra of $\mathrm{CsPbCl}_{x} \mathrm{Br}_{3-x} @ \mathrm{Ag}$ hybrid nanocrystals obtained by reacting $\mathrm{CsPbBr}_{3}$ nanocrystals with $\mathrm{AgCl}$ for $0-24 \mathrm{~h}$. The inset in (c) shows photographs of the $\mathrm{CsPBBr}_{3}$ nanocrystals and $\mathrm{CsPbCl}_{x} \mathrm{Br}_{3-x} @ \mathrm{Ag}$ hybrid nanocrystals at a reaction time of $24 \mathrm{~h}$ under $365 \mathrm{~nm}$ UV light irradiation. The enhancement is mainly attributed to the enhanced absorbance of UV or blue light by the Ag-induced plasmonic near-field effect. Adapted with permission ${ }^{142}$. Copyright 2017, RSC. e Left image: schematic of the Au/p-NiO/CsPbBr 3 QD/MgZnO/Au NP/n-ZnO/n+ ${ }^{+}-\mathrm{GaN}$ heterostructure LED. Right image: cross-sectional SEM image of the $\mathrm{NiO} / \mathrm{CsPbBr}_{3} \mathrm{QD} / \mathrm{MgZnO} / \mathrm{Au} \mathrm{NP} / \mathrm{ZnO}$ coaxial NWs. (f) EL spectra of the plasmonic PeLED with Au NP decoration and the reference device without Au NPs captured at $8.0 \mathrm{~V}$. The insets present the corresponding emission photographs of an emitting unit $\left(2 \times 2 \mathrm{~mm}^{2}\right)$. The electroluminescence enhancement is associated with the increased spontaneous emission rate and improved internal QY induced by exciton-LSPR coupling. Adapted with permission ${ }^{150}$. Copyright 2018, Wiley.

Ag NPs need to be optimized so that the positive effect is stronger than the negative effect. Xu et al. reported a perovskite blue LED with Au NPs embedded in PEDOT: PSS $^{143}$. The fluorescence intensity was improved by 3.8 times. The maximum luminance and EQE reached $\sim 1110$ $\mathrm{cd} / \mathrm{m}^{2}$ and $1.64 \%$, respectively.
Liu et al. placed disordered Au NRs underneath a film of $\mathrm{CsPbBr}_{3} \mathrm{NCs}^{144}$ and studied their emission performance. By using femtosecond laser pulses at $800 \mathrm{~nm}$, amplified spontaneous emission (ASE) at approximately $523 \mathrm{~nm}$ was excited by two-photon pumping. A narrow peak with a linewidth of $5 \mathrm{~nm}$ appeared with the pump fluence 
reaching a low threshold of $0.65 \mathrm{~mJ} / \mathrm{cm}^{2}$, owing to the plasmonic effects of $\mathrm{Au}$ NRs improving the emission transition rate. et al. precipitated Ag NPs and $\mathrm{CsPbBr}_{3}$ perovskite QDs in borosilicate glass by the conventional melt-quenching method ${ }^{145}$. Part of $\mathrm{Pb}^{2+}$ was replaced by $\mathrm{Ag}^{+}$in the $\mathrm{CsPbBr}_{3}$ QDs, and the remaining $\mathrm{Ag}^{+}$, which did not react with $\mathrm{Pb}^{2+}$, was converted into $\mathrm{Ag}$ NPs. These Ag NPs enhanced the PL by 5 times for the $\mathrm{CsPbBr}_{3}$ QD-doped glasses, which was mainly due to the enhanced optical absorption and LSPR of the Ag NPs. Yun et al. proposed stretchable plasmonic templates of $\mathrm{Au}$ and $\mathrm{Au} / \mathrm{SiO}_{2} \mathrm{NPs}$ embedded in polymer to improve the luminescence of $\mathrm{CsPbBr}_{3} \mathrm{NCs}^{146}$. The charge carrier imbalance due to hole trapping in the perovskite LEDs with naked NPs could be prevented by embedding $\mathrm{Au}$ or $\mathrm{Au} / \mathrm{SiO}_{2} \mathrm{NPs}$ in the polymer. The luminescence of the perovskite $\mathrm{NC}$ film on the template with $\mathrm{Au} / \mathrm{SiO}_{2} \mathrm{NPs}$ increased by $54 \%$ compared with that of the perovskite $\mathrm{NC}$ film on the reference (polymer film without metal NPs). The luminescence enhancement of $\mathrm{Au} / \mathrm{SiO}_{2} \mathrm{NPs}$ was ascribed to coupling to the LSPRs of the $\mathrm{Au} /$ $\mathrm{SiO}_{2}$ NPs.

$\mathrm{Li}$ et al. studied the performance of perovskite QD (PQD) films coupled to Ag NW networks (NWKs) with a spacer of polyvinyl alcohol (PVA) between the PQD film and NWKs ${ }^{147}$. Compared with bare quartz, the PVA substrate significantly enhanced the emission intensity but reduced the emission rate of $\mathrm{PQD}$ excitons. When NWK was introduced, the emission intensity and rate exhibited a maximum of 6-fold (average 3.4-fold) and 2.4-fold (average 1.9-fold) increases, respectively, due to efficient NWK-PQD coupling. They further demonstrated a Purcell-enhanced emission rate in a hybrid structure of $\mathrm{PQDs}$ with plasmonic crystals at room temperature ${ }^{148}$. Colloidal PQDs, Ag nanocubes, and PVP have been incorporated together into a device by chemical assembly. The PL enhancement can be modified in both the steady-state fluorescence and time-resolved measurements by changing the PVP spacer thickness and Ag nanocube surface density. The total fluorescence intensity and emission rate demonstrate 3.5 -fold and 4.5fold enhancements, respectively.

A plasmonic $\mathrm{Au} \mathrm{NR} / \mathrm{CsPbBr} \mathrm{Pb}_{3}$ QD film was fabricated via spin-coating ${ }^{149}$. The $\mathrm{PL}$ intensity of the $\mathrm{Au}$ NR/ $\mathrm{CsPbBr}_{3}$ QD film demonstrated a 2-fold enhancement compared to that of the $\mathrm{CsPbBr}_{3}$ QD film without $\mathrm{Au}$ NRs. The $\mathrm{CsPbBr}_{3}$ QD film exhibited a lifetime decrease from $6.15 \mathrm{~ns}$ to $4.78 \mathrm{~ns}$ after incorporating Au nanorods. The Au nanorods triggered an LSPR of approximately 500 $\mathrm{nm}$, which enhanced the emission intensity of the $\mathrm{CsPbBr}_{3}$ QD film. Shi et al. fabricated a $\mathrm{Au} / \mathrm{p}-\mathrm{NiO} /$ $\mathrm{CsPbBr}_{3} \mathrm{QD} / \mathrm{MgZnO} / \mathrm{Au} \mathrm{NP} / \mathrm{n}-\mathrm{ZnO} / \mathrm{n}^{+}-\mathrm{GaN}$ heterostructure LED (Fig. 6e, f) ${ }^{150}$. The emission was enhanced by 1.55 times after embedding Au NPs into the device.
The luminance, EQE, and current efficiency of the optimized plasmonic-perovskite LED reached $10206 \mathrm{~cd} / \mathrm{m}^{2}$, $\sim 4.626 \%$, and $8.736 \mathrm{~cd} / \mathrm{A}$, respectively. The electroluminescence performance was enhanced due to the increased spontaneous emission rate and improved internal QY, which was induced by the coupling between the exciton and LSPR.

Plasmonic films can also enhance the PL. Chen et al. placed $\mathrm{CsPbBr}_{3}$ nanoplatelets between $\mathrm{Ag}$ NWs and a $\mathrm{Ag}$ film. Strong biexciton emission was induced with continuous-wave excitation at room temperature ${ }^{151}$. The exciton occupancy for generating biexcitons was reduced by $\sim 10^{6}$ times in the Ag NW-Ag film nanogaps. The nonlinear Fano resonance between biexcitons and plasmonic cavity modes was responsible for the great enhancement in biexciton emissions. Adamo et al. coated $\mathrm{MAPbI}_{3}$ on a Au slit film ${ }^{152}$. The luminescence intensity was enhanced by more than 10 times compared with that of pure $\mathrm{MAPbI}_{3}$, while the luminescence lifetime demonstrated a nearly 3-fold decrease. The improvement in luminescence intensity was attributed to the significantly enhanced Purcell effect with an enhancement factor of more than 300 .

Plasmonic NPs and films with Au and Ag materials have been used in perovskite LEDs and have achieved impressive PL and EQE enhancements. It is expected that more nanostructures and different material combinations with stronger plasmonic enhancement will be explored for perovskite LEDs. The impact of the plasmonic nanostructure on the crystal quality and stability should also be studied more thoroughly.

\section{Plasmonic-perovskite lasers}

The properties of plasmonic-based perovskite lasers, such as the threshold, wavelength, polarization, and laser emission characteristics, can be facilely controlled by the rational design of the coupling between perovskites and plasmonic nanostructures. Plasmonic lasers with a low mode volume have been realized by coupling the perovskite with plasmonic NPs and films. This progress advances the development of high-performance perovskite micro/nanolasers.

NPs have been used for plasmonic-perovskite lasers. Wang et al. demonstrated plasmonic-enhanced laser emissions from all-inorganic lead-halide perovskites ${ }^{153}$. Uniform Al NPs were deposited onto the top surface of $\mathrm{CsPbBr}_{3}$ perovskites by electron beam evaporation. The resonance of the $\mathrm{Al} \mathrm{NPs}$ was tuned to the emission wavelength range of $\mathrm{CsPBrr}_{3}$ to enhance the PL. A $20 \%$ reduction in the thresholds of whispering gallery mode lasers in $\mathrm{CsPbBr}_{3}$ perovskite microrods was observed. The plasmonic effects enhanced the output intensities of perovskite microlasers by more than an order of magnitude. Huang et al. demonstrated the plasmonic-perovskite laser 

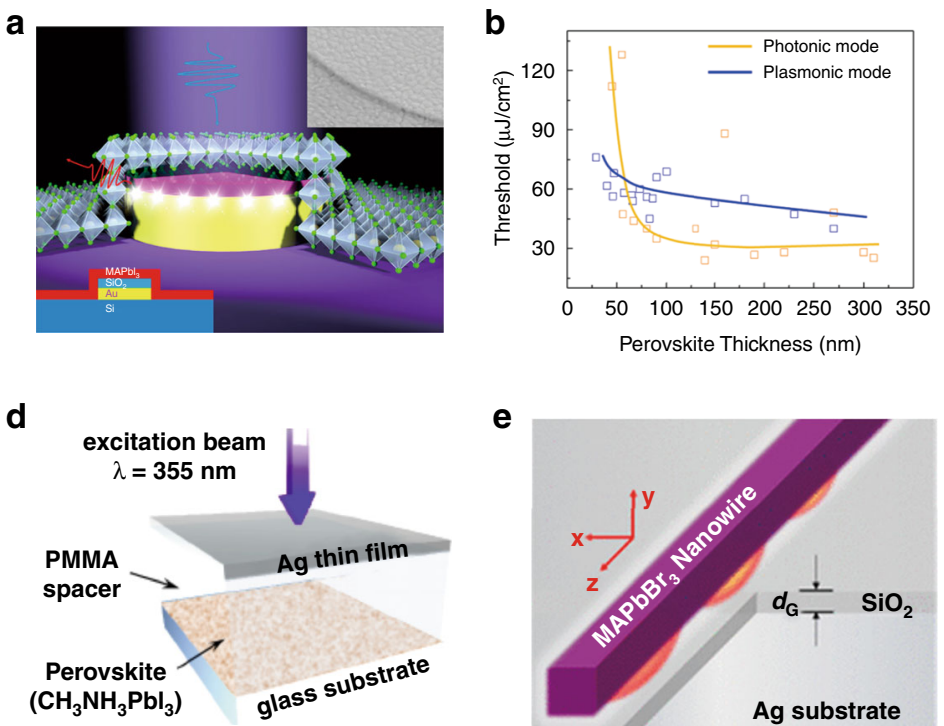

e

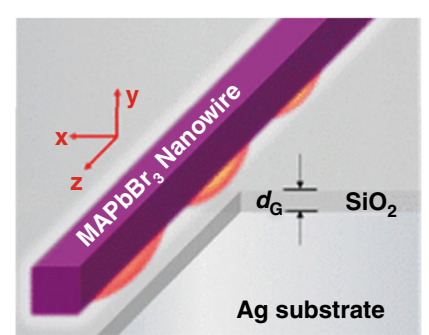

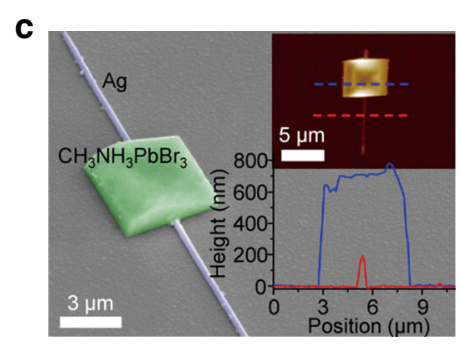

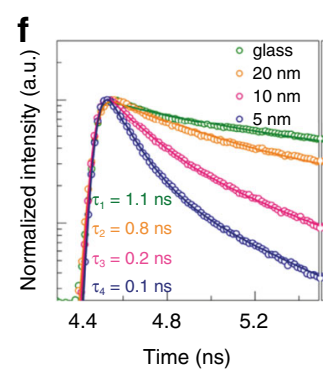

Fig. 7 Plasmonic-perovskite lasers. a Schematic of a spaser (surface plasmon amplification by stimulated emission of radiation) composed of a $\mathrm{MAPb}_{3} \mathrm{NP} / \mathrm{SiO}_{2} / \mathrm{Ag}$ plasmonic laser. Inset: SEM image of perovskite NPs lying on $\mathrm{Au} / \mathrm{SiO}_{2}$ microdisks. In contrast to previous reports, here, the spasers are determined by the boundary of Au patterns instead of the crystal facets of $\mathrm{MAPbX}_{3}$ nanosheets. As a result, whispering gallery mode-based circular spasers and spaser arrays are successfully realized by patterning the Au substrate into circles and gratings, respectively. $\mathbf{b}$ Lasing threshold of plasmonic and photonic lasers versus perovskite thickness; the solid lines are used for guidance. Only the hybrid plasmonic mode exists below a perovskite thickness of $40 \mathrm{~nm}$. Adapted with permission ${ }^{154}$. Copyright 2018, ACS. c SEM image of a perovskite/Ag heterostructure. Insets: AFM image and corresponding cross-sectional profiles of a typical heterostructure. The perovskite crystals can serve as both the gain medium and the optical resonant cavity for low-threshold lasing. Adapted with permission ${ }^{155}$. Copyright 2016, ACS. d Schematic layer structure of the perovskites capped with a dielectric PMMA spacer and a Ag thin film. Not only can perovskite be protected from hydrolysis but also the lasing thresholds can be greatly reduced due to the plasmonic effect. Adapted with permission ${ }^{161}$. Copyright 2016, OSA. e Schematic of a hybrid plasmonic microcavity structure. A $\mathrm{MAPbBr}_{3}$ nanowire sits on $\mathrm{SiO}_{2} / \mathrm{Ag}$ substrate $(\sim 50 \pm 1 \mathrm{~nm})$ with a spacer layer of $\mathrm{SiO}_{2}\left(d_{\mathrm{G}} \approx 5-20 \pm 1 \mathrm{~nm}\right)$. The exciton-photon coupling strength is enhanced by $\sim 35 \%$ on average, which is mainly attributed to surface plasmon-induced localized excitation field redistribution. $\mathbf{f}$ Time-resolved PL spectra of $\mathrm{MAPbBr}_{3} \mathrm{NWs}$ sitting on glass and $\mathrm{SiO}_{2} / \mathrm{Ag}$ substrates with $\mathrm{SiO}_{2}$ thicknesses of 20, 10, and $5 \mathrm{~nm}$. Adapted with permission ${ }^{162}$. Copyright 2018, ACS.

by placing $\mathrm{MAPb} X_{3}$ perovskite nanosheets on Au patterns with a $10 \mathrm{~nm} \mathrm{SiO}{ }_{2}$ spacer (Fig. 7a) ${ }^{154}$. Whispering gallery modes were successfully excited by Au substrates with circle and grating patterns. Resonances within the hybrid plasmonic nanolaser could be precisely controlled by the shape and size of the bottom Au patterns instead of the top semiconductors, revealing the substrate-control concept. The standard wavelength deviation was as small as $0.3 \mathrm{~nm}$. The emission wavelengths could be tuned within a $200 \mathrm{~nm}$ wavelength span by varying the stoichiometry of the perovskite. Hybrid plasmonic nanolasers were generated, leading to lasing even with a small perovskite thickness $(<40 \mathrm{~nm})$ (Fig. 7b). Li et al. used Ag NWs as nucleation centers for growing perovskites, constructing a perovskite/Ag heterostructure (Fig. 7c) ${ }^{155}$. Low-threshold lasing was achieved because the perovskite crystals not only were a gain medium but also formed an optical resonant cavity. The lasing modes could be modulated by tuning the plasmonic effects on the perovskite microcavities. Wu et al. placed a polymethyl methacrylate (PMMA) spacer layer and a Au NR-doped PMMA top layer on perovskite thin films ${ }^{156}$. The ASE threshold and intensity of the perovskite with Au NRs demonstrated an $\sim 36 \%$ decrease and 13.9-fold increase, respectively, compared to those of perovskite films without Au NRs. The enhancement was ascribed to both the contribution of surface passivation due to PMMA and the plasmonic effects of $\mathrm{Au}$ NRs. Chen et al. introduced a detachable $\mathrm{Al}$ $\mathrm{NP}$ substrate to enhance $\mathrm{MAPbBr} \mathrm{r}_{3}$ perovskite lasing performance ${ }^{157}$. The lasing threshold and stimulated emission of the Al-MAPbBr 3 hybrid system were dramatically reduced by more than $27 \%$ and improved by 10 times, respectively, due to the plasmonic effects of the Al NPs. Hsieh et al. fabricated perovskite lasers based on the configuration of $\mathrm{Ag}$ nanocube/CsPbBr 3 quantum dot/ $\mathrm{Al}_{2} \mathrm{O}_{3} / \mathrm{Au}$. Cs $\mathrm{PbBr}_{3}$ quantum dots were placed in the nanogap between the $\mathrm{Ag}$ nanocube and $\mathrm{Au}$ film. The threshold decreased to $1.9 \mathrm{~W} / \mathrm{cm}^{2}$ at $120 \mathrm{~K}$, which was due to the ultrasmall mode volume and significant Purcell enhancement at the corner of the nanocavity inside the gap $^{158}$. Lu et al. reported upconversion lasing based on the configuration of a single $\mathrm{MAPbBr}_{3}$ perovskite nanocrystal 
(PNC) $/ \mathrm{Al}_{2} \mathrm{O}_{3} / \mathrm{TiN} /$ silicon $^{159}$. TiN served as a resonanceadjustable plasmonic nanocavity and generated strong localized electromagnetic fields. Both the two-photon pumping rate and emission rate were enhanced by the plasmonic resonances and enabled the upconversion lasing action. The lasing threshold decreased to $10 \mu \mathrm{J} / \mathrm{cm}^{2}$, which was at least 3 orders of magnitude smaller than the threshold of the reference sample (a PNC on silicon). Huang et al. demonstrated a hybrid plasmonic surface lattice resonance (SLR) laser consisting of a $\mathrm{MAPbBr}_{3}$ perovskite thin film on an Ag NP array ${ }^{160}$. The SLR mode could be tuned to couple the green light emission of the perovskite material by the design of the NP parameters, thus enhancing the lasing performance. The pumping threshold was approximately $0.54 \mu \mathrm{W}$, and the linewidth of the lasing signal was only $0.28 \mathrm{~nm}$.

The effects of plasmonic films on the perovskite laser performance have also been studied. Lasing performance is enhanced in lead-halide perovskites covered with PMMA and Ag (Fig. 7d) ${ }^{161}$. The PMMA layer can provide protection from hydrolysis in the ambient environment. The lasing threshold can be reduced by more than two orders of magnitude compared with that of bare perovskite because of the strong exciton-plasmon coupling between the Ag and perovskite films. Shang et al. reported strong exciton-photon interactions in $\mathrm{MAPbBr}_{3}$ perovskite NWs (Fig. 7e $)^{162}$. Characteristic anticrossing behaviors were observed near the exciton resonance in the hybrid perovskite $\mathrm{NW} / \mathrm{SiO}_{2} / \mathrm{Ag}$ cavity at room temperature, which indicated a Rabi splitting energy up to $\sim 564 \mathrm{meV}$. The exciton-photon coupling strength was improved by $\sim 35 \%$ on average, which was mainly ascribed to the near-field redistribution induced by the plasmonic resonances. Compared with that of the NW directly on the glass substrate, the spontaneous emission rate was enhanced by $11,5.5$, and 1.4 times with $\mathrm{SiO}_{2}$ thicknesses of 5,10 , and $20 \mathrm{~nm}$, respectively (Fig. $7 \mathrm{f}$ ). Yu et al. reported a plasmonic-perovskite laser by placing perovskite NWs on an Ag substrate, where an insulating spacer layer was inserted ${ }^{163}$. Relatively low threshold $\left(13.5 \mu \mathrm{J} / \mathrm{cm}^{2}\right)$ operation was obtained under ambient conditions, and the devices worked well even at temperatures up to $43.6{ }^{\circ} \mathrm{C}$. Wu et al. realized plasmonic lasing with a threshold down to $\sim 6.5 \mu \mathrm{J} / \mathrm{cm}^{2}$ at room temperature using $\mathrm{CsPbBr}_{3} \mathrm{NW}$-based plasmonic devi$\operatorname{ces}^{164}$. Polarization-sensitive pump responses and highly polarized emission parallel to the NW axis were observed due to the plasmonic modes. The Purcell effect reduced the radiative recombination lifetime of $\mathrm{CsPBr}_{3}$ NWs by a factor of $\sim 6.14$. The lasing threshold of the plasmonic device increased as the NW length became larger. This may make plasmonic devices smaller and more integrated than conventional photonic devices. S. Yang et al. demonstrated an ultrafast nanolaser based on a triple-layer structure of perovskite $/ \mathrm{MgF}_{2} / \mathrm{Ag}$ film produced with a scalable solution method ${ }^{165}$. The emission dynamics were accelerated by the strong plasmonic confinement of the laser modes due to the coupling between the perovskite emission and SPs. Single-mode lasing behavior was clearly observed at room temperature. The plasmonic-perovskite laser not only reduced the device size but also significantly promoted the emission efficiency. Wang et al. studied the laser performance of a single $\mathrm{CH}_{3} \mathrm{NH}_{3} \mathrm{PbBr}_{3}$ nanowire on a silver film without any insulating layer ${ }^{166}$. The nonhybridized plasmonic nanowire lasers exhibited ultrafast lasing dynamics with an approximately 1.9 ps decay rate and a 1 ps peak response time. The low threshold was due to the ultraflat single-crystal silver films and high-quality single-crystal perovskite nanowires.

Plasmonic-perovskite lasers show great potential for use in integrated optics due to their small size, low threshold, and controlled wavelength and polarization. The Purcell effect can be enhanced by plasmonic resonances, which increases the light-matter interaction and emission intensity while reducing the emission lifetime, thus enabling faster lasers and faster LEDs. Future challenges include the ability to achieve efficient carrier injection and the realization of an electrically pumped plasmonic-perovskite laser. In addition, smarter nanostructures and plasmonic-perovskite interface designs are needed to further reduce the device size and footprint, as well as reduce the lasing threshold.

\section{Plasmonic-perovskite sensors \\ Plasmonic-perovskite photodetectors}

The performance of PDs can be improved by plasmonic nanostructures because they enhance the coupling between incident light and the semiconducting materials that generate photocurrents. In the work of Dong et al., $\mathrm{Au}$ NCs were incorporated into $\mathrm{Cs} \mathrm{PbBr}_{3} \mathrm{NC}$ visible-light $\mathrm{PDs}^{167}$. The PDs with Au NCs exhibited a higher sensitivity with an on/off ratio that was more than one order of magnitude larger than that of the initial device at a $2 \mathrm{~V}$ bias under $532 \mathrm{~nm}$ laser illumination $\left(4.65 \mathrm{~mW} / \mathrm{cm}^{2}\right)$. Moreover, the photocurrent increased by $238 \%$ (from the original $245.6 \mu \mathrm{A}$ to $831.1 \mu \mathrm{A}$ ) with the incorporation of Au NCs due to the near-field plasmonic enhancement. B. $\mathrm{Du}$ et al. fabricated an organic-inorganic hybrid perovskite PD with periodic Au nanosquares (Fig. 8a-d) ${ }^{168}$. The maximum EQE of this PD was up to $65 \%$, which was 2.5-fold higher than that of a similar hybrid perovskite placed on a usual $\mathrm{Si} / \mathrm{SiO}_{2}$ substrate without $\mathrm{Au}$ nanosquares. The large, localized $E$-field induced by the SPR of $\mathrm{Au}$ nanostructures was responsible for the enhancement. With a configuration similar to that in Fig. 8a, Gu et al. studied the effect of $\mathrm{Au}$ nanotriangles on $\mathrm{PD}$ performance ${ }^{169}$. The EQE of $\mathrm{MAPbI}_{3}$-Au was $\sim 3$ times higher 

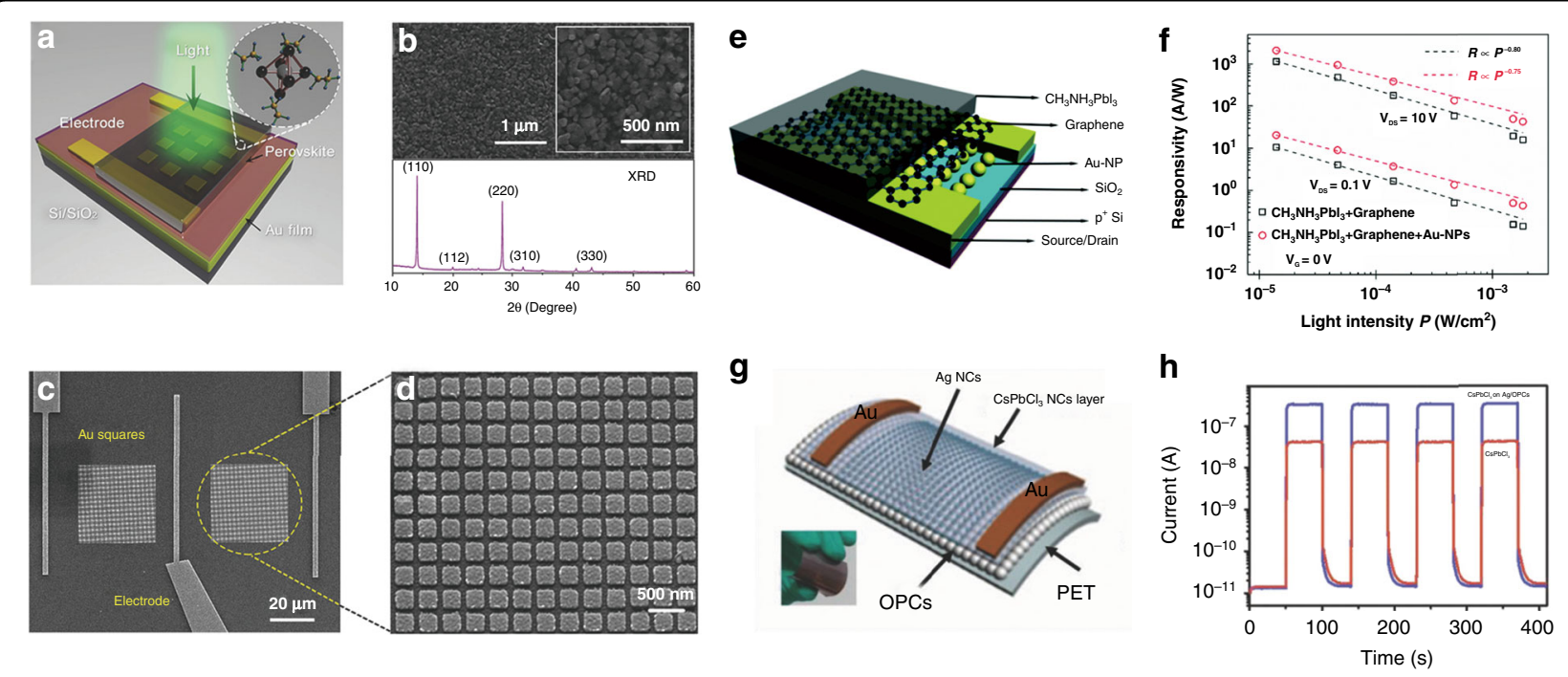

Fig. 8 Plasmonic-perovskite photodetectors. a-d Organic-inorganic hybrid perovskite PD on arrays of Au nanostructures. The maximum EQE of this PD is as high as approximately $65 \%, 2.5$ times that on a usual $\mathrm{Si} / \mathrm{SiO}_{2}$ substrate without Au nanostructures, which is due to the large, localized E-field induced by the SPR. a Schematic of the device on a plasmonic substrate. $\mathbf{b}$ SEM images of the plasmonic substrate. $\mathbf{c}$ The dependence of the photocurrent on the incident laser power for the device on $\mathrm{Si}_{/} \mathrm{SiO}_{2}$ and plasmonic substrates at zero bias. $\mathbf{d}$ EQE of devices on $\mathrm{Si} / \mathrm{SiO}_{2}$ and plasmonic substrates. Adapted with permission ${ }^{168}$. Copyright 2019, Wiley. e, f Graphene/MAPbl 3 hybrid PDs integrated with Au NPs. e Schematic of the Au NP/ graphene/ $\mathrm{CH}_{3} \mathrm{NH}_{3} \mathrm{Pbl}_{3}$ hybrid PDs. $\mathbf{f}$ Responsivities versus light intensity at different $V_{\mathrm{DS}}$ biases. The dependencies of the responsivities on the light intensity are fitted with power functions with the listed fitting parameters. Compared with pristine graphene-MAPbl ${ }_{3}$ devices, this device has two times higher photoresponsivity and a faster photoresponse speed. This enhancement can be attributed to the improved light absorption in the perovskite layer due to the plasmonic effect of the Au NPs. Adapted with permission ${ }^{170}$. Copyright 2016, RSC. g, h CsPbCl $/ 2 / \mathrm{Ag} / \mathrm{OPC}$ hybrid PDs. (g) Schematic of $\mathrm{CsPbCl}_{3}$ on Ag/OPC PDs. h On-off switching properties measured under $365 \mathrm{~nm}$ light illumination $\left(10 \mathrm{~mW} / \mathrm{cm}^{2}\right)$ at a bias of $3 \mathrm{~V}$. The photocurrent of $\mathrm{CsPbCl}_{3} \mathrm{PD}$ with $\mathrm{Ag} / \mathrm{OPC}$ is $682 \%$ higher than that of $\mathrm{CsPbCl}_{3} \mathrm{PD}$ without $\mathrm{Ag} / \mathrm{OPC}$. Adapted with permission ${ }^{176}$. Copyright 2018, Wiley.

than that of the $\mathrm{MAPbI}_{3}$ film without Au nanotriangles. The photoresponsivity of the $\mathrm{MAPbI}_{3}$-Au-based $\mathrm{PD}$ was $51 \mathrm{~mA} / \mathrm{W}$ at $10 \mathrm{~V}$ with a power density of $1.5 \mathrm{~W} / \mathrm{cm}^{2}$ and an EQE of $12.6 \%$ for an illumination wavelength of 450 $\mathrm{nm}$. Au NPs were integrated into the graphene/MAPbI hybrid PDs (Fig. 8e, f) ${ }^{170}$. The device with Au NPs exhibited a 2-fold higher photoresponsivity and a faster photoresponse speed compared with those of pristine graphene- $\mathrm{MAPbI}_{3}$ devices. Plasmon-enhanced light harvesting was improved on the perovskite-graphene interface, which led to a higher PD operation speed and carrier extraction efficiency than devices without Au NPs. Ghosh et al. reported an air-processed high-performance selfpowered hybrid perovskite PD whose ETL was embedded with plasmonic Ag NPs ${ }^{171}$. At zero bias, the ETL-free Ag NP-perovskite hybrid device showed a $\sim 45 \%$ enhanced responsivity and operated with a $\sim 3$ times faster photoresponse than the pristine device. The enhancements were due to a series of positive effects introduced by the plasmonic Ag NPs, such as enhanced light absorption, hot electron generation, and improved charge extraction and transport.

Ji et al. showed a quasi-2D perovskite $\mathrm{PD}$ with $\mathrm{SiO}_{2}$ coated AuAg-alloyed nanoprisms (AuAg-NPrisms@SiO ${ }_{2}$ ) between the PEDOT:PSS and perovskite layers ${ }^{172}$.
The PDs with AuAg-NPrisms@SiO ${ }_{2}$ exhibited a high EQE of $1670 \%$ at a low driving voltage of $-0.3 \mathrm{~V}$. Their responsivity and detectivity reached $7.15 \mathrm{~A} / \mathrm{W}$ and $3.2 \times$ $10^{13}$ Jones, which were $51.2 \%$ and $68.4 \%$ higher than those of PDs without NPs. The enhancement was because the AuAg-NPrisms@SiO ${ }_{2}$ led to high-quality crystalline quasi2D perovskite films and stronger light absorption in the active layer, accelerating exciton dissociation at the interface and facilitating charge transport. Li et al. reported a $\mathrm{PD}$ composed of perovskite/Al NP/anodic aluminum oxide $(\mathrm{AAO})^{173}$. The hybrid plasmonic-perovskite nanostructures showed a 43-fold increase in the photocurrent, which was due to the enhanced $E$-fields around the Au NPs. Wang et al. used plasmonic bowtie nanoantenna arrays to boost the performance of a perovskite $\mathrm{PD}^{174}$. The photoresponse and detectivity of the plasmonic-perovskite PD increased by $\sim 2962 \%$ and more than 30 times compared with those of a $\mathrm{Si} / \mathrm{SiO}_{2}$-based conventional PD, respectively. Electric fields were greatly enhanced around the bowtie nanoantenna arrays due to the LSPR effect, which led to the improvement. La et al. realized a perovskite PD by embedding assembled Au NPs in the PEDOT:PSS layer ${ }^{175}$. Charge extraction/transport and optical absorption were enhanced by the assembled $\mathrm{Au}$ NPs due to the good conductivity and plasmonic 
resonance of the Au NPs. The responsivity, noise equivalent power (NEP), and response speed of the perovskite with $\mathrm{Au}$ NPs were $\sim 2063 \mathrm{~A} / \mathrm{W}, \sim 1.93 \times 10^{-14} \mathrm{~W} / \mathrm{Hz}^{1 / 2}$, and $\sim 300 \mathrm{~ns}$, respectively, which were approximately 4.1-, 15.6-, and 2.8-fold increases, respectively, compared with those of a perovskite PD without NPs.

$\mathrm{Ag}$ films composed of $\mathrm{Ag} \mathrm{NCs}$ and $\mathrm{CsPbCl}_{3} \mathrm{NCs}$ were used for high-performance flexible ultraviolet photodetectors (Fig. 8g, h) ${ }^{176}$. The luminescent intensity enhancement of the $\mathrm{CsPbCl}_{3} \mathrm{NCs}$ showed a more than 150-fold improvement with an estimated emission efficiency of $51.5 \%$. The improvement was due to the combined excitation and emission field enhancement. The $\mathrm{CsPbCl}_{3} / \mathrm{Ag} /$ opal photonic crystal (OPC) hybrids exhibited a dark current of $10^{-11} \mathrm{~A}$, detectivity of $9 \times$ $10^{14}$ Jones, response time of $28 \mathrm{~ms}$, and response linewidth of $30 \mathrm{~nm}$. This performance was claimed to exceed that of commercial silicon PDs at the time that the paper was published.

By integrating plasmonic nanostructures with perovskites, enhanced light absorption can be attained, which opens the door for high-performance photodetectors. Moreover, plasmonic metals can also act as electrodes and perform carrier extraction without increasing the complexity of the configuration. The challenge will be to avoid carrier tunneling between metals and perovskite and simultaneously increase the conductivity. Different combinations of halide perovskite materials and plasmonic nanostructures should be explored to maximize photodetector performance across the desired wavelength span.

\section{Plasmonic-perovskite optical sensors}

Recently, halide perovskites have been explored for applications as refractometric sensors, surface-enhanced Raman scattering (SERS), and biologic sensors. Elshorbagy et al. theoretically reported a perovskite refractometric sensor with an extruded array of high aspect-ratio dielectric pyramids, which excited SPRs at its front surface by grating coupling with the metal surface ${ }^{177}$. The generated photocurrent acted as the sensing signal, where selective absorption with a spectral response narrower than $1 \mathrm{~nm}$ was achieved. Without relying on the spectral acquisition scheme, the perovskite device refractometric sensor used an optoelectronic interrogation method. The device showed considerable sensing performance in the refraction index range between 1.0 and 1.1. The figure of merit (FOM) could reach 1005 $\mathrm{RIU}^{-1}$. In addition, the perovskite refractometric sensor also possessed the advantages of a simple signal acquisition procedure and low cost.

Qiao et al. demonstrated a superficial-layer-enhanced Raman scattering (SLERS) technique for detecting species that were noninteractive with Raman substrates. The configuration consisted of elongated tetrahexahedral
Au NP (ETHH Au NP) arrays covered with a superficial $\mathrm{CH}_{3} \mathrm{NH}_{3} \mathrm{PbBr}_{3}$ film (Fig. 9a, b) ${ }^{178}$. These ETHH NP arrays provided uniform and high-density SERS hotspots, and the perovskite film acted as a dielectric media to slow the attenuation of the electromagnetic evanescent wave of the hotspots, which made SLERS occur in the superficial layer rather than just on the surface of the ETHH Au NPs. Zeng et al. presented a sensor based on a hybrid metasurface consisting of $2 \mathrm{D}$ perovskites and metal thin films ${ }^{179}$. The device showed a sharp optical phase change and a GoosHänchen shift around the wavelength of the SPP (Fig. 9c). The Goos-Hänchen $(\mathrm{GH})$ shift exhibited a sensitivity of $900,000 \mu \mathrm{m} / \mathrm{RIU}$, which was more than 4 orders of magnitude higher than that of a pure Ag sensing substrate (800 $\mu \mathrm{m} / \mathrm{RIU})$ (Fig. 9d). When silver nanogrooves were used (Fig. 9e), coupling between the LSPR and SPP waves led to a deeper dip in the angular spectra, implying a further improvement in the detection sensitivity. Such 2D perovskite-based metasurfaces show promise for use as ultrasensitive in situ biosensors.

Halide perovskites have not been widely studied as sensors; thus, reports of plasmonic-perovskite sensors are rarer. Nevertheless, plasmonic sensors are a mature technology with numerous chemical, biological and medical detection applications. The combination of plasmonics and perovskite technologies will break new boundaries in sensitivity and pave the way for a truly compact and integrated optical sensor platform. We also envision the use of plasmonic-perovskite sensors to detect other physical and environmental parameters in different technological domains.

\section{Other plasmonic-perovskite applications}

The performance of perovskites in photocatalysis can also be enhanced by plasmonic NPs. Ag NPs have been synthesized on perovskite orthorhombic $\mathrm{KNbO}_{3} \mathrm{NWs}$ by facile photoreduction ${ }^{180} . \mathrm{Ag} / \mathrm{KNbO}_{3}$ nanocomposites with different Ag contents (0.4-2.8 wt\%) have been used to monitor the degradation of aqueous rhodamine $\mathrm{B}$ under $\mathrm{UV}$ and visible light. As the Ag content is increased, the UV-induced photoreactivity first increases, reaching a maximum at a Ag content of $\sim 1.7 \mathrm{wt} \%$, and then decreases. The maximum photoreactivity is $\sim 13$-fold higher than that of pristine $\mathrm{KNbO}_{3}$ without Ag NPs. In contrast, visible light-induced photoreactivity shows a monotonic positive correlation with $\mathrm{Ag}$ contents from 0.4 wt\% to 2.8 wt\%. Saris et al. used $\mathrm{CsPbBr}_{3} \quad \mathrm{NC} / \mathrm{AlO}_{x}$ composite films with Ag NCs to drive chemical reactions based on the coupling between perovskite and Ag NCs (Fig. 10a, b) ${ }^{181}$. It was found that energy migrated from the perovskite NCs toward the Ag NCs. This energy migration was utilized to boost plasmon-mediated methylene blue desorption, in which coupling to the perovskite NCs enhanced the spatial and spectral absorption of the 
a

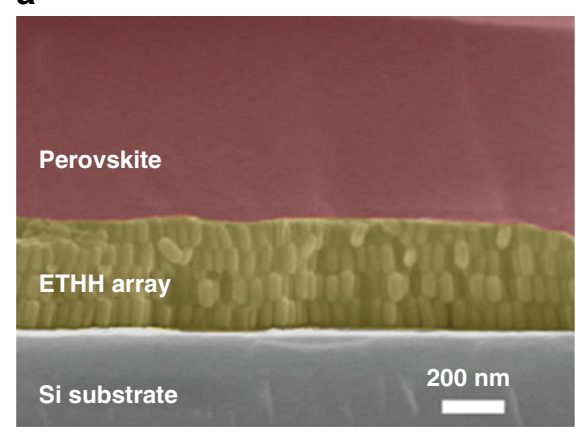

b

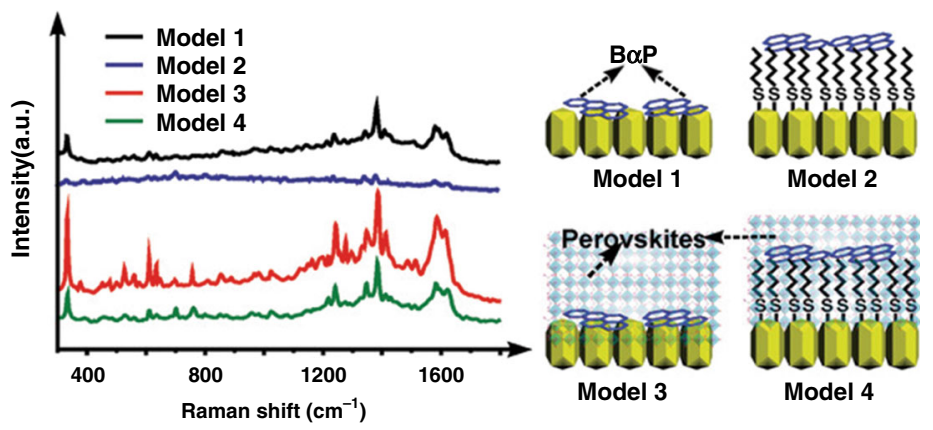

C

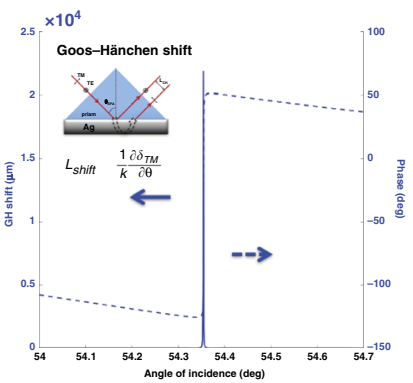

d

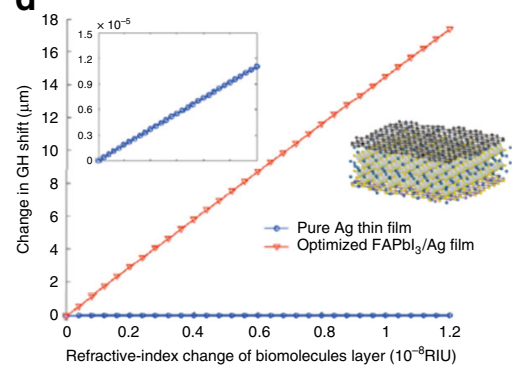

$\mathbf{e}_{0.04}$

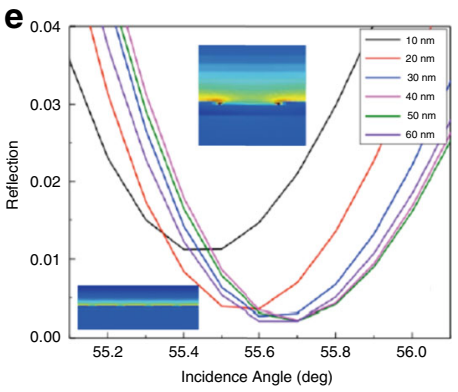

Fig. 9 Plasmonic-perovskite optical sensors. a, b Superficial-layer-enhanced Raman scattering (SLERS) technique based on covering elongated tetrahexahedral (ETHH) Au NP arrays with a $\mathrm{CH}_{3} \mathrm{NH}_{3} \mathrm{PbBr}_{3}$ film. Noninteracting analytes, such as benzo[a]pyrene (BaP) that has chronic toxicity and can easily adhere to human skin or muscles to cause carcinogenesis, is detected. a Cross-sectional SEM images of the perovskite film on ETHH NP arrays. b Raman spectra of BaP in four different models without (Model 1 and Model 2) and with (Model 3 and Model 4) the perovskite. BaP is segregated from the ETHH arrays by n-alkyl thiols in Model 2 and Model 4. The Raman signals are significantly enhanced with the perovskite because the perovskite film, as a dielectric medium, slows the attenuation of the electromagnetic evanescent wave and the LSPR decay is suppressed along the vertical direction away from the nanostructured surface. Adapted with permission ${ }^{178}$. Copyright 2019, Wiley. c Optical phase and GH shift as a function of the angle of incidence near the SPP resonance angle of $54.36^{\circ}$. The sensing substrate consists of a bilayer $\mathrm{FAPbl}_{3}$ on a $45-\mathrm{nm}$ silver thin film. $\mathbf{d}$ Simulations showing that a plasmonic sensor based on graphene/FAPbl $3 / \mathrm{hBN} / \mathrm{Ag}$ can detect small refractive index changes on the order of $10^{-8} \mathrm{RIU}$, which is 4 orders of magnitude more sensitive than plasmonic sensors based on a simple Au substrate. The presence of $2 \mathrm{D}$ perovskite layers of a suitable thickness can lead to deeper SPP resonance dips, which correspond to a sharper optical phase change and a larger GH shift. e Simulated angular reflectance spectra for a device consisting of bilayer $\mathrm{FAPb}_{3}$ on silver nanogrooves with various groove widths. The insets show that the enhanced near fields lead to deeper spectral dips. Adapted with permission ${ }^{214}$. Copyright 2020, MPDI.

chemical reaction. Chanana et al. proposed a triple-layered structure of $\left(\mathrm{C}_{6} \mathrm{H}_{5} \mathrm{C}_{2} \mathrm{H}_{4} \mathrm{NH}_{3}\right)_{2}\left(\mathrm{CH}_{3} \mathrm{NH}_{3}\right)_{\mathrm{n}-1}\left(\mathrm{PbI}_{4}\right)_{\mathrm{n}} /$ $\mathrm{MAPbI}_{3} / \mathrm{Al}$ hole arrays/silicon substrate (Fig. 10c) for the photoinduced modulation of $\mathrm{THz}$ resonance ${ }^{182}$. The sample with $n=1$ showed a distinct $\mathrm{THz}$ transmission peak, exhibiting no optical intensity dependence when illuminated at $700 \mathrm{~nm}$ (Fig. 10d), since that wavelength did not correspond to photogenerated excitons in the perovskite layer. When the samples were illuminated in the wavelength range where excitons were formed, the increased $\mathrm{THz}$ absorption within the holes led to lower overall $\mathrm{THz}$ transmission with increasing optical intensity (Fig. 10e-g). This work indicated that the plasmonic effect could be tuned by the perovskite. Zhou et al. reported a polarization-dependent modulation based on hybrid perovskite plasmon-induced transparency (PIT) ${ }^{183}$. A $\mathrm{MAPbI}_{3}$ film was coated on the PIT acting as a photoactive medium. Even with ultralow laser fluence pumping, a significant reduction in PTI transmission was observed for the two polarizations at 0.86 and $1.12 \mathrm{THz}$ with a recovery time of $561 \mathrm{ps}$, demonstrating the high sensitivity and ultrafast modulation speed of the device.

\section{Conclusions and outlook}

In recent years, researchers have demonstrated the use of plasmonics to enhance the performance of perovskitebased solar cells, light emitters, photodetectors, sensors, and many other applications. Regarding plasmonic PSCs, the majority of studies are based on NPs because NPs can be synthesized and easily incorporated into devices. We observed several trends from the vast amount of literature on NP-assisted PSCs that we examined: (1) the PCE of PSCs can be significantly enhanced by plasmonic NPs; (2) PSCs with plasmonic NPs incorporated at the perovskite$\mathrm{m}-\mathrm{TiO}_{2}$ interface or simultaneously at multiple positions can potentially achieve the largest PCE enhancement; (3) NPs with a core-shell structure, shape modification, and dimer show stronger effects than that of simple spherical 


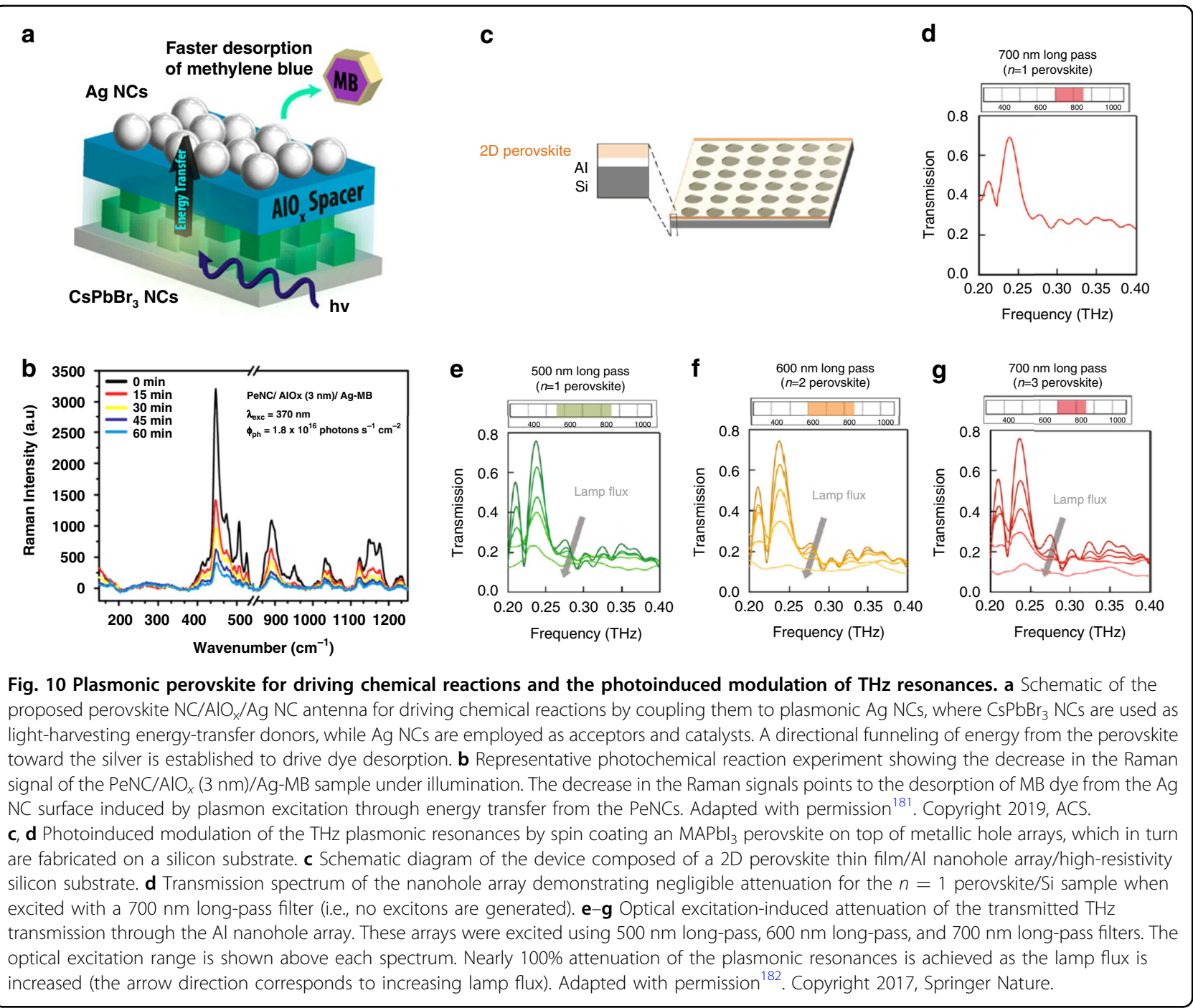

NPs; (4) alloyed NPs show stronger enhancement than that of a single material; and (5) the size and concentration have different effects on the current, voltage, and FF; thus, they need to be optimized carefully. Based on these observations, we infer that alloyed core-shell nanorods with sharp tips and sub-10 $\mathrm{nm}$ gaps positioned at the perovskite- $\mathrm{m}-\mathrm{TiO}_{2}$ interface are promising for substantially increasing PV performance. The size and concentration may be optimized when $<50 \mathrm{~nm}$ and $<2 \mathrm{wt} \%$, respectively. Plasmonic (nanostructured) films have not been widely used in PSCs because their fabrication is usually more complicated, and they can introduce more loss. Nevertheless, plasmonic films can still be promising for high PV performance, as plasmonic films provide a larger penetration depth of SPPs, which can exert a larger influence on $E$-field enhancement, conductivity, and other factors. In addition to increasing light absorption and photocurrent generation, plasmonic NPs and films can also enhance the light emission of perovskites, which benefits the applications of LEDs and lasers. The Purcell effect can be enhanced by plasmonic resonances, which increase the light-matter interaction and emission intensity while reducing the emission lifetime, thus enabling faster LEDs and lasers. Plasmonic NPs and films also significantly improve the performance of perovskites used for optical detection and sensing, photocatalysis, and $\mathrm{THz}$ modulation. Simulations and experiments demonstrate that these enhancements are mainly attributed to the plasmonic-optical (near-field absorption and far-field scattering) effect.

Although the combination of perovskite and plasmonics has progressed rapidly, there are still some challenges to overcome. First, the quality, stability, and reliability of halide perovskites remain major problems for perovskiterelated applications. In addition to efforts to improve the intrinsic properties of perovskites, it would be interesting 
to study changes in the quality, stability, and reliability after the addition of plasmonic nanostructures in the vicinity of perovskites, especially the use of NPs to improve these properties. Second, novel plasmonic nanostructures can be further explored and optimized to meet specific application demands ${ }^{184-186}$, through the control of the wavelength, light angles and polarizations, optical confinement, hotspot positions, coupling and proximity effects. Perovskite materials and compositions that can seamlessly integrate with plasmonic nanostructures can be identified based on realistic fabrication conditions and constraints, band alignment, etc. ${ }^{12,36,187}$. Light manipulation in plasmonic nanostructures and adaptive perovskite materials will lead to more applications and the further maximization of their performance. Third, the mechanism behind plasmonic-perovskite interactions should be studied in greater depth. Although macroscopic enhancement mechanisms are well understood, other intricate phenomena (such as optical quenching, carrier tunneling, and quantum effects) at the nanoscale remains unanswered. In addition, the separation of the various optical and electrical effects is still an open question along with determining the exact contribution from each effect. Fourth, machine learning (ML) is a powerful tool for designing and screening perovskite materials ${ }^{188,189}$, as well as for inverse design in nanophotonics ${ }^{190-192}$. There are many opportunities to apply ML toward plasmonic perovskites, such as to assist in the determination of the material, structure, and device architecture of plasmonic-perovskite systems for improved performance. All these challenges and opportunities encourage deeper theoretical analysis and further experimental studies in the years to come.

\section{Acknowledgements \\ B.A., Z.F., and Z.J.W. acknowledge funding support from the President's Excellence Fund (X-Grant) and the TAMU start-up grant. B.A. was partially supported by the Natural Science Foundation of Chongqing of China (cstc2020jcyj-msxmX0614), the Fundamental Research Funds for the Central Universities (2021CDJQY-010), the Open Foundation of Defense Key Disciplines Lab of Novel Micro-Nano Devices and System Technology, and the Open Foundation of Chongqing University Key Lab of Optoelectronic Materials and Engineering.}

\section{Author details}

'Department of Aerospace Engineering, Texas A\&M University, College Station, TX 77843, USA. ${ }^{2}$ School of Microelectronics and Communication Engineering, Chongqing University, 400044 Chongqing, P.R. China. ${ }^{3}$ Chongqing Key Laboratory of Bioperception \& Intelligent Information Processing, 400044 Chongqing, P.R. China. ${ }^{4}$ Department of Materials Science and Engineering, Texas A\&M University, College Station, TX 77843, USA

\section{Competing interests}

The authors declare no competing interests.

Received: 5 July 2021 Revised: 6 October 2021 Accepted: 28 October 2021 Published online: 12 January 2022

\section{References}

1. Dubey, A. et al. A strategic review on processing routes towards highly efficient perovskite solar cells. J. Mater. Chem. A 6, 2406-2431 (2018).

2. Ansari, M. I. H., Qurashi, A. \& Nazeeruddin, M. K. Frontiers, opportunities, and challenges in perovskite solar cells: a critical review. J. Photochem. Photobiol., C. 35, 1-24 (2018).

3. Liu, S. et al. A review on additives for halide perovskite solar cells. Adv. Energy Mater. 10, 1902492 (2019).

4. Fu, Y. P. et al. Metal halide perovskite nanostructures for optoelectronic applications and the study of physical properties. Nat. Rev. Mater. 4, 169-188 (2019).

5. Buchanan, M. Perovskite fever. Nat. Phys. 16, 996-996 (2020).

6. Zhu, M., Niesner, D. \& Zhu, X. Y. Perovskite fever: absorbing and emitting light. Abstr. Pap. Am. Chem. Soc. 251 (2016).

7. Cheng, X., Yang, S., Cao, B., Tao, X. \& Chen, Z. Single crystal perovskite solar cells: development and perspectives. Adv. Funct. Mater. 30, 1905021 (2019).

8. Bai, S. et al. Planar perovskite solar cells with long-term stability using ionic liquid additives. Nature 571, 245-250 (2019).

9. Ono, L. K., Juarez-Perez, E. J. \& Qi, Y. Progress on perovskite materials and solar cells with mixed cations and halide anions. ACS Appl. Mater. Interfaces $\mathbf{9}$, 30197-30246 (2017).

10. Jung, E. H. et al. Efficient, stable and scalable perovskite solar cells using poly (3-hexylthiophene). Nature 567, 511-515 (2019).

11. Lin, K. B. et al. Perovskite light-emitting diodes with external quantum efficiency exceeding 20 per cent. Nature 562, 245-248 (2018).

12. Cao, Y. et al. Perovskite light-emitting diodes based on spontaneously formed submicrometre-scale structures. Nature 562, 249-253 (2018).

13. Van, Le,Q., Jang, H. W. \& Kim, S. Y. Recent advances toward high-efficiency halide perovskite light-emitting diodes: review and perspective. Small Methods 2, 1700419 (2018).

14. Matsushima, T. et al. High performance from extraordinarily thick organic light-emitting diodes. Nature 572, 502-506 (2019).

15. Schlaus, A. P. et al. How lasing happens in $\mathrm{CsPbBr}_{3}$ perovskite nanowires. Nat. Commun. 10, 8 (2019).

16. Jia, Y. F., Kerner, R. A., Grede, A. J., Rand, B. P. \& Giebink, N. C. Continuous-wave lasing in an organic-inorganic lead halide perovskite semiconductor. Nat. Photonics 11, 784-788 (2017).

17. Veldhuis, S. A. et al. Perovskite materials for light-emitting diodes and lasers. Adv. Mater. 28, 6804-6834 (2016).

18. Yang, Z. Q. et al. High-performance single-crystalline perovskite thin-film photodetector. Adv. Mater. 30, 7 (2018).

19. Tian, W., Zhou, H. P. \& Li, L. Hybrid organic-inorganic perovskite photodetectors. Small 13, 22 (2017).

20. Ahmadi, M., Wu, T. \& Hu, B. A review on organic-inorganic halide perovskite photodetectors: device engineering and fundamental physics. Adv. Mater. 29, 24 (2017).

21. Mathews, l. et al. Self-powered sensors enabled by wide-bandgap perovskite indoor photovoltaic cells. Adv. Funct. Mater. 29, 7 (2019).

22. Hsu, K. C., Fang, T. H., Hsiao, Y. J. \& Wu, P. C. Response and characteristics of $\mathrm{TiO}_{2}$ /perovskite heterojunctions for $\mathrm{CO}$ gas sensors. J. Alloy. Compd. 794, 576-584 (2019).

23. Huang, H. W. et al. C(sp(3))-H bond activation by perovskite solar photocatalyst cell. ACS Energy Lett. 4, 203-208 (2019).

24. Qian, X. X. et al. Perovskite cesium lead bromide quantum dots: a new efficient photocatalyst for degrading antibiotic residues in organic system. J. Clean. Prod. 249, 8 (2020).

25. Dai, Y. T., Poidevin, C., Ochoa-Hernandez, C., Auer, A. A. \& Tuysuz, H. A supported bismuth halide perovskite photocatalyst for selective aliphatic and aromatic C-H bond activation. Angew. Chem. Int. Ed. 59, 5788-5796 (2019).

26. Bera, K. P. et al. Graphene sandwich stable perovskite quantum-dot lightemissive ultrasensitive and ultrafast broadband vertical phototransistors. ACS Nano 13, 12540-12552 (2019).

27. Kim, B. S. Y., Hikita, Y., Yajima, T. \& Hwang, H. Y. Heteroepitaxial vertical perovskite hot-electron transistors down to the monolayer limit. Nat. Commun. 10, 5312 (2019).

28. $\mathrm{He}, \mathrm{G}$. et al. Silver modified $\mathrm{Ba}_{1}-\mathrm{XCO}_{0.7} \mathrm{Fe}_{0.2} \mathrm{Nb}_{0.1} \mathrm{O}_{3}$-delta perovskite performing as a cathodic catalyst of intermediate temperature solid oxide fuel cells. ACS Appl. Mater. Interfaces 12, 9421-9433 (2020)

29. Zhang, $\mathrm{H}$., Chen, G. \& Tang, L. Cubic perovskite-type $\mathrm{Sm}_{0.3} \mathrm{Sr}_{0.7} \mathrm{Nb}_{0.08} \mathrm{CO}_{0.92} \mathrm{O}_{3}$ delta as a novel cathode material for intermediate temperature solid oxide fuel cells. Chem. Commun. 56, 1361-1364 (2020). 
30. Zhu, Y. Y. et al. Bromine vacancy redistribution and metallic-ion-migrationinduced air-stable resistive switching behavior in all-inorganic perovskite cspbbr $_{3}$ film-based memory device. Adv. Electron. Mater. 6, 1900754 (2019).

31. Zou, C., Zheng, J. J., Chang, C., Majumdar, A. \& Lin, L. Y. Nonvolatile rewritable photomemory arrays based on reversible phase-change perovskite for optical information storage. Adv. Opt. Mater. 7, 1900558 (2019).

32. Belykh, V. V. et al. Coherent spin dynamics of electrons and holes in $\mathrm{CsPbBr}_{3}$ perovskite crystals. Nat. Commun. 10, 673 (2019).

33. Wang, $K$. et al. Spin-polarized electronic transport through ferromagnet/ organic-inorganic hybrid perovskite spinterfaces at room temperature. Adv. Mater. Interfaces 6, 1900718 (2019).

34. Kojima, A., Teshima, K., Shirai, Y. \& Miyasaka, T. Organometal halide perovskites as visible-light sensitizers for photovoltaic cells. J. Am. Chem. Soc. 131, 6050-6051 (2009)

35. Kim, H. S. et al. Lead iodide perovskite sensitized all-solid-state submicron thin film mesoscopic solar cell with efficiency exceeding 9\%. Sci. Rep.-Uk 2, 591 (2012).

36. Hu, C., Li, M. Y., Qiu, J. S. \& Sun, Y. P. Design and fabrication of carbon dots for energy conversion and storage. Chem. Soc. Rev. 48, 2315-2337 (2019).

37. Braly, I. L. et al. Hybrid perovskite films approaching the radiative limit with over 90\% photoluminescence quantum efficiency. Nat. Photonics 12 355-361 (2018).

38. Mao, L., Wu, Y., Stoumpos, C. C., Wasielewski, M. R. \& Kanatzidis, M. G. Whitelight emission and structural distortion in new corrugated two-dimensional lead bromide perovskites. J. Am. Chem. Soc. 139, 5210-5215 (2017).

39. Dohner, E. R., Jaffe, A., Bradshaw, L. R. \& Karunadasa, H. I. Intrinsic white-light emission from layered hybrid perovskites. J. Am. Chem. Soc. 136, 13154-13157 (2014)

40. Kim, Y. H. et al. Comprehensive defect suppression in perovskite nanocrystals for high-efficiency light-emitting diodes. Nat. Photonics 15, 148-155 (2021).

41. Di, D. W. et al. High-performance light-emitting diodes based on carbenemetal-amides. Science 356, 159-163 (2017)

42. Dai, X. L. et al. Solution-processed, high-performance light-emitting diodes based on quantum dots. Nature 515, 96-99 (2014).

43. Cook, D. \& Nash, F. Gain-induced guiding and astigmatic output beam of GaAs lasers. J. Appl. Phys. 46, 1660-1672 (1975).

44. Xing, G. et al. Low-temperature solution-processed wavelength-tunable perovskites for lasing. Nat. Mater. 13, 476 (2014).

45. Zhu, $H$. et al. Lead halide perovskite nanowire lasers with low lasing thresholds and high quality factors. Nat. Mater. 14, 636-642 (2015).

46. Zhang, N. et al. Postsynthetic and selective control of lead halide perovskite microlasers. J. Phys. Chem. Lett. 7, 3886-3891 (2016).

47. Liao, Q. et al. Perovskite microdisk microlasers self-assembled from solution Adv. Mater. 27, 3405-3410 (2015).

48. Wang, K. et al. Formation of single-mode laser in transverse plane of perovskite microwire via micromanipulation. Opt. Lett. 41, 555-558 (2016).

49. Zhang, $\mathrm{N}$. et al. Highly reproducible organometallic halide perovskite microdevices based on top-down lithography. Adv. Mater. 29, 1606205 (2017)

50. Chen, S. et al. A photonic crystal laser from solution based organo-lead iodide perovskite thin films. ACS Nano 10, 3959-3967 (2016).

51. Pourdavoud, N. et al. Photonic nanostructures patterned by thermal nanoimprint directly into organo-metal halide perovskites. Adv. Mater. 29 1605003 (2017).

52. Jia, Y., Kerner, R. A., Grede, A. J., Rand, B. P. \& Giebink, N. C. Continuous-wave lasing in an organic-inorganic lead halide perovskite semiconductor. Nat. Photonics 11, 784-788 (2017).

53. Liu, X., Zhang, Q., Xiong, Q. \& Sum, T. C. Tailoring the lasing modes in semiconductor nanowire cavities using intrinsic self-absorption. Nano Lett. 13, 1080-1085 (2013).

54. Liu, X. et al. Periodic organic-inorganic halide perovskite microplatelet arrays on silicon substrates for room-temperature lasing. Adv. Sci. 3, 1600137 (2016).

55. Gu, Z. et al. Direct-writing multifunctional perovskite single crystal arrays by inkjet printing. Small 13, 1603217 (2017).

56. Deng, $\mathrm{H}$. et al. Flexible and semitransparent organolead triiodide perovskite network photodetector arrays with high stability. Nano Lett. 15, 7963-7969 (2015).

57. Chen, K. \& Tüysüz, H. Morphology-controlled synthesis of organometal halide perovskite inverse opals. Angew. Chem. Int. Ed. 54, 13806-13810 (2015).

58. Zhuo, S., Zhang, J., Shi, Y., Huang, Y. \& Zhang, B. Self-template-directed synthesis of porous perovskite nanowires at room temperature for high- performance visible-light photodetectors. Angew. Chem. Int. Ed. $\mathbf{5 4}$ 5693-5696 (2015).

59. Miao, J. \& Zhang, F. Recent progress on highly sensitive perovskite photodetectors. J. Mater. Chem. C. 7, 1741-1791 (2019).

60. Yu, X., Tsao, H. N., Zhang, Z. \& Gao, P. Miscellaneous and perspicacious: Hybrid halide perovskite materials based photodetectors and sensors. Adv. Opt. Mater. 8, 2001095 (2020)

61. Xia, H.-R., Li, J., Sun, W.-T. \& Peng, L.-M. Organohalide lead perovskite based photodetectors with much enhanced performance. Chem. Commun. 50, 13695-13697 (2014).

62. Lin, Y., Lin, G., Sun, B. \& Guo, X. Nanocrystalline perovskite hybrid photodetectors with high performance in almost every figure of merit. Adv. Funct. Mater. 28, 1705589 (2018).

63. Kim, J. H. \& Kim, S.-H. Sub-second pyridine gas detection using a organometal halide perovskite functional dye. Dyes Pigm. 134, 198-202 (2016).

64. Xu, W. et al. An ultrasensitive and reversible fluorescence sensor of humidity using perovskite $\mathrm{CH}_{3} \mathrm{NH}_{3} \mathrm{PbBr}_{3}$. J. Mater. Chem. C 4, 9651-9655 (2016).

65. Niu, Y. et al. Aggregation-induced emission features of organometal halide perovskites and their fluorescence probe applications. Adv. Opt. Mater. $\mathbf{3}$ 112-119 (2015).

66. Saraf, R., Pu, L. \& Maheshwari, V. A light harvesting, self-powered monolith tactile sensor based on electric field induced effects in mapbi ${ }_{3}$ perovskite. Adv. Mater. 30, 1705778 (2018).

67. Yoo, D. et al. Origin of the stability and transition from anionic to cationic surface ligand passivation of all-inorganic cesium lead halide perovskite nanocrystals. J. Phys. Chem. Lett. 11, 652-658 (2020).

68. Yao, D. et al. Dimensionality-controlled surface passivation for enhancing performance and stability of perovskite solar cells via triethylenetetramine vapor. ACS Appl. Mater. Interfaces 12, 6651-6661 (2020).

69. Wang, W. H. \& Qi, L. M. Light management with patterned micro- and nanostructure arrays for photocatalysis, photovoltaics, and optoelectronic and optical devices. Adv. Funct. Mater. 29, 1807275 (2019).

70. Chan, K., Wright, M., Elumalai, N., Uddin, A. \& Pillai, S. Plasmonics in organic and perovskite solar cells: Optical and electrical effects. Adv. Opt. Mater. $\mathbf{5}$ 1600698 (2017).

71. Jang, Y. H. et al. Plasmonic solar cells: From rational design to mechanism overview. Chem. Rev. 116, 14982-15034 (2016).

72. Chen, J. D., Jin, T. Y., Li, Y. Q. \& Tang, J. X. Recent progress of light manipulation strategies in organic and perovskite solar cells. Nanoscale 11, 18517-18536 (2019).

73. Ozbay, E. Plasmonics: merging photonics and electronics at nanoscale dimensions. Science 311, 189-193 (2006).

74. Atwater, H. A. The promise of plasmonics. Sci. Am. 296, 56-63 (2007).

75. Kasani, S., Curtin, K. \& Wu, N. Q. A review of 2D and 3D plasmonic nanostructure array patterns: fabrication, tight management and sensing applications. Nanophotonics 8, 2065-2089 (2019).

76. Ghahremanirad, E., Olyaee, S. \& Hedayati, M. The influence of embedded plasmonic nanostructures on the optical absorption of perovskite solar cells. Photonics 6, 37 (2019).

77. Lee, D. S. et al. Self-position of au nps in perovskite solar cells: Optical and electrical contribution. ACS Appl. Mater. Interfaces 8, 449-454 (2016).

78. Chang, S. H., Lin, K. F., Chiang, C. H., Chen, S. H. \& Wu, C. G. Plasmonic structure enhanced exciton generation at the interface between the perovskite absorber and copper nanoparticles. Sci. World J. 2014, 128414 (2014).

79. Carretero-Palacios, S., Calvo, M. E. \& Miguez, H. Absorption enhancement in organic-inorganic halide perovskite films with embedded plasmonic gold nanoparticles. J. Phys. Chem. C 119, 18635-18640 (2015).

80. Pathak, N. K. \& Sharma, R. P. Study of broadband tunable properties of surface plasmon resonances of noble metal nanoparticles using mie scattering theory: plasmonic perovskite interaction. Plasmonics 11, 713-719 (2016).

81. Chaiyachate, P. \& Dasri, T. Optical absorption and scattering properties of the active layer of perovskite solar cells incorporated silver nanoparticles. Orient. J. Chem. 33, 807-813 (2017).

82. Yue, L. Y., Yan, B., Attridge, M. \& Wang, Z. B. Light absorption in perovskite solar cell: fundamentals and plasmonic enhancement of infrared band absorption. Sol. Energy 124, 143-152 (2016).

83. Pathak, N. K. \& Sharma, R. P. Study of surface plasmon resonance of core-shell nanogeometry under the influence of perovskite dielectric environment: Electrostatic approximation. AIP Conf. Proc. 1731, 050063 (2016). 
84. Hu, Z. S., Ma, T. L. \& Hayase, S. Interparticle coupling effect of silver-gold heterodimer to enhance light harvesting in ultrathin perovskite solar cell. J. Photon. Energy 8, 015502 (2018)

85. Heidarzadeh, H. Comprehensive investigation of core-shell dimer nanoparticles size, distance and thicknesses on performance of a hybrid organicinorganic halide perovskite solar cell. Mater. Res. Express 5, 036208 (2018).

86. Ghahremanirad, E. et al. Improving the performance of perovskite solar cells using kesterite mesostructure and plasmonic network. Sol. Energy 169, 498-504 (2018).

87. Kim, K. \& Lee, S. Detailed balance analysis of plasmonic metamaterial perovskite solar cells. Opt. Express 27, A1241-A1260 (2019).

88. Hajjiah, A., Kandas, I. \& Shehata, N. Efficiency enhancement of perovskite solar cells with plasmonic nanoparticles: a simulation study. Materials 11, 1626 (2018).

89. Ghahremanirad, E., Olyaee, S., Nejand, B. A., Ahmadi, V. \& Abedi, K. Hexagonal array of mesoscopic htm-based perovskite solar cell with embedded plasmonic nanoparticles. Phys. Status Solidi B 255, 1700291 (2018).

90. Cai, B., Peng, Y., Cheng, Y. B. \& Gu, M. 4-fold photocurrent enhancement in ultrathin nanoplasmonic perovskite solar cells. Opt. Express 23, A1700-1706 (2015).

91. Mohebpour, M. A., Saffari, M., Soleimani, H. R. \& Tagani, M. B. High performance of mixed halide perovskite solar cells: Role of halogen atom and plasmonic nanoparticles on the ideal current density of cell. Phys. E 97 282-289 (2018).

92. Pathak, H., Ji, A., Pathak, N. K. \& Sharma, R. P. Resonant broadband field enhancement in cylindrical plasmonic structure surrounded by perovskite environment. Plasmonics 12, 1511-1522 (2017).

93. Wang, G. et al. Wide-angle polarization-free plasmon-enhanced light absorption in perovskite films using silver nanowires. Opt. Express $\mathbf{2 5}$ 3594-3604 (2017).

94. Abdelraouf, O. A. M., Shaker, A. \& Allam, N. K. Novel design of plasmonic and dielectric antireflection coatings to enhance the efficiency of perovskite solar cells. Sol. Energy 174, 803-814 (2018).

95. Abdelraouf, O. A. M., Shaker, A. \& Allam, N. K. All dielectric and plasmonic cross-grating metasurface for efficient perovskite solar cells. Metamaterials $X$ 10671, 1067120 (2018)

96. Perrakis, G. et al. Efficient and environmental-friendly perovskite solar cells via embedding plasmonic nanoparticles: an optical simulation study on realistic device architectures. Opt. Express 27, 31144-31163 (2019).

97. Wang, Y. et al. Enhanced efficiency of perovskite solar cells by using core-

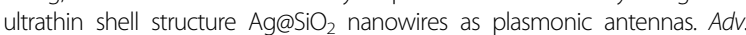
Electron. Mater. 3, 1700169 (2017).

98. Guo, Y., He, X., Liu, X., Li, X. \& Kang, L. One-step implementation of plasmon enhancement and solvent annealing effects for air-processed high-efficiency perovskite solar cells. J. Mater. Chem. A 6, 24036-24044 (2018).

99. Chandrasekhar, P. S. et al. Higher efficiency perovskite solar cells using Au@SiO2 core-shell nanoparticles. Sustain. Energ. Fuels 2, 2260-2267 (2018).

100. Shalan, A. E. et al. Versatile plasmonic-effects at the interface of inverted perovskite solar cells. Nanoscale 9, 1229-1236 (2017).

101. Wu, R. et al. Prominent efficiency enhancement in perovskite solar cells employing silica-coated gold nanorods. J. Phys. Chem. C 120, 6996-7004 (2016).

102. Luo, Q. et al. Plasmonic effects of metallic nanoparticles on enhancing performance of perovskite solar cells. ACS Appl. Mater. Interfaces 9, 34821-34832 (2017)

103. Song, S. et al. Optically tunable plasmonic two-dimensional Ag quantum dot arrays for optimal light absorption in polymer solar cells. J. Phys. Chem. C 121, 17569-17576 (2017).

104. Chen, X. \& Gu, M. Hole blocking layer-free perovskite solar cells with high efficiencies and stabilities by integrating subwavelength-sized plasmonic alloy nanoparticles. ACS Appl. Energy Mater. 2, 2094-2103 (2019).

105. Zhang, W. et al. Enhancement of perovskite-based solar cells employing core-shell metal nanoparticles. Nano Lett. 13, 4505-4510 (2013).

106. Ali, A., Kang, J. H., Seo, J. H. \& Walker, B. Effect of plasmonic Ag nanoparticles on the performance of inverted perovskite solar cells. Adv. Eng. Mater. 22, 1900976 (2019).

107. Yang, D. et al. Correlations of optical absorption, charge trapping, and surface roughness of $\mathrm{TiO}_{2}$ photoanode layer loaded with neat ag-nps for efficient perovskite solar cells. ACS Appl. Mater. Interfaces 8, 21522-21530 (2016).

108. Batmunkh, M. et al. Plasmonic gold nanostars incorporated into highefficiency perovskite solar cells. ChemSusChem 10, 3750-3753 (2017).
109. Ma, C. et al. Plasmonic-enhanced light harvesting and perovskite solar cell performance using Au biometric dimers with broadband structural darkness. Sol. RRL 3, 1900138 (2019).

110. Cheng, Y. et al. Considerably enhanced perovskite solar cells via the introduction of metallic nanostructures. J. Mater. Chem. A 5, 6515-6521 (2017).

111. Sun, Z. Q. et al. Enhanced p-i-n type perovskite solar cells by doping AuAg@AuAg core-shell alloy nanocrystals into PEDOT:PSS layer. Org. Electron. 52 309-316 (2018).

112. Wang, B. et al. Enhancing the photovoltaic performance of perovskite solar cells using plasmonic Au@Pt@Au core-shell nanoparticles. Nanomaterials 9 1263 (2019).

113. Cho, J. S., Jang, W., Park, S., Kim, B. K. \& Wang, D. H. Thermally stable propanethiol-ligand exchanged Ag nanoparticles for enhanced dispersion in perovskite solar cells via an effective incorporation method. J. Ind. Eng. Chem. 61, 71-77 (2018).

114. Tang, M. Y. et al. Fine-tuning the metallic core-shell nanostructures for plasmonic perovskite solar cells. Appl. Phys. Lett. 109, 183901 (2016).

115. Saliba, M. et al. Plasmonic-induced photon recycling in metal halide perovskite solar cells. Adv. Funct. Mater. 25, 5038-5046 (2015).

116. Fu, N. Q. et al. Panchromatic thin perovskite solar cells with broadband plasmonic absorption enhancement and efficient light scattering management by Au@Ag core-shell nanocuboids. Nano Energy 41, 654-664 (2017).

117. Liu, Y. et al. Enhancement of photocurrent in an ultra-thin perovskite solar cell by Ag nanoparticles deposited at low temperature. RSC Adv. 7, 1206-1214 (2017).

118. Fan, R. D. et al. Tailored Au@TiO2 nanostructures for the plasmonic effect in planar perovskite solar cells. J. Mater. Chem. A 5, 12034-12042 (2017).

119. Mali, S. S., Shim, C. S., Kim, H., Patil, P. S. \& Hong, C. K. In situ processed gold nanoparticle-embedded $\mathrm{TiO} 2$ nanofibers enabling plasmonic perovskite solar cells to exceed 14\% conversion efficiency. Nanoscale 8, 2664-2677 (2016).

120. Higgins, M. et al. Enhanced reproducibility of planar perovskite solar cells by fullerene doping with silver nanoparticles. J. Appl. Phys. 124, 065306 (2018).

121. Ginting, R. T. et al. Plasmonic effect of gold nanostars in highly efficient organic and perovskite solar cells. ACS Appl. Mater. Interfaces 9, 36111-36118 (2017).

122. Panigrahi, S. et al. Mapping the space charge carrier dynamics in plasmonbased perovskite solar cells. J. Mater. Chem. A 7, 19811-19819 (2019).

123. Choy, W. C. \& Ren, X. Plasmon-electrical effects on organic solar cells by incorporation of metal nanostructures. IEEE J. Sel. Top. Quantum Electron. 22 1-9 (2015).

124. Liu, $\mathrm{C}$. et al. Improved performance of perovskite solar cells with a $\mathrm{TiO}_{2} / \mathrm{MoO}_{3}$ core/shell nanoparticles doped PEDOT: PSS hole-transporter. Org. Electron. 33, 221-226 (2016)

125. Wang, Z. K. et al. Improved hole interfacial layer for planar perovskite solar cells' with efficiency exceeding 15\%. ACS Appl. Mater. Interfaces 7, 9645-9651 (2015).

126. Pathak, N. K., Chander, N., Komarala, V. K. \& Sharma, R. P. Plasmonic perovskite solar cells utilizing Au@SiO 2 core-shell nanoparticles. Plasmonics 12, 237-244 (2017).

127. Zhang, C. X. et al. Efficient perovskite solar cells by combination use of Au nanoparticles and insulating metal oxide. Nanoscale 9, 2852-2864 (2017).

128. Garcia-Vidal, F. J., Martin-Moreno, L., Ebbesen, T. \& Kuipers, L. Light passing through subwavelength apertures. Rev. Mod. Phys. 82, 729 (2010).

129. Ebbesen, T. W., Lezec, H. J., Ghaemi, H., Thio, T. \& Wolff, P. A. Extraordinary optical transmission through sub-wavelength hole arrays. Nature 391, 667-669 (1998).

130. Ai, B., Yu, Y., Möhwald, H., Wang, L. \& Zhang, G. Resonant optical transmission through topologically continuous films. ACS Nano 8, 1566-1575 (2014).

131. Ai, B., Wang, L., Möhwald, H., Yu, Y. \& Zhang, G. Confined surface plasmon sensors based on strongly coupled disk-in-volcano arrays. Nanoscale 7 2317-2324 (2015).

132. Ai, B., Yu, Y., Möhwald, H., Zhang, G. \& Yang, B. Plasmonic films based on colloidal lithography. Adv. Colloid Interface Sci. 206, 5-16 (2014).

133. Barnes, W. L. Surface plasmon-polariton length scales: a route to subwavelength optics. J. Opt. A: Pure Appl. Opt. 8, S87-S93 (2006).

134. Chen, $M$. et al. Profiling light absorption enhancement in two-dimensional photonic-structured perovskite solar cells. IEEE J. Photovolt. 7, 1324-1328 (2017).

135. Chen, M., Wang, Y. \& Zhang, Y. Enhanced light absorption of textured perovskite solar cells employing two-dimensional nanoarrays. J. Photon. Energy $\mathbf{9}$ 037001 (2019). 
136. Abdelraouf, O. A. M., Shaker, A. \& Allam, N. K. Front dielectric and back plasmonic wire grating for efficient light trapping in perovskite solar cells. Opt. Mater. 86, 311-317 (2018).

137. Long, M. Z. et al. Ultrathin efficient perovskite solar cells employing a periodic structure of a composite hole conductor for elevated plasmonic light harvesting and hole collection. Nanoscale 8, 6290-6299 (2016).

138. Wei, J. et al. Enhanced light harvesting in perovskite solar cells by a bioinspired nanostructured back electrode. Adv. Energy Mater. 7, 1700492 (2017)

139. Kim, G. M. \& Tatsuma, T. Photocurrent enhancement of perovskite solar cells at the absorption edge by electrode-coupled plasmons of silver nanocubes. J. Phys. Chem. C 121, 11693-11699 (2017).

140. Zhang, $X$. et al. Plasmonic perovskite light-emitting diodes based on the Ag-CsPbBr 3 System. ACS Appl. Mater. Interfaces 9, 4926-4931 (2017).

141. Mokkath, J. H. Dopant-induced localized light absorption in $\mathrm{CsPbX} 3(X=\mathrm{Cl}$, $\mathrm{Br}$, I) perovskite quantum dots. N. J. Chem. 43, 18268-18276 (2019).

142. Ye, S., Yu, M. H., Yan, W., Song, J. \& Qu, J. L. Enhanced photoluminescence of CsPbBr3@Ag hybrid perovskite quantum dots. J. Mater. Chem. C 5, 8187-8193 (2017).

143. $\mathrm{Xu}, \mathrm{T}$. F. et al. High-performance blue perovskite light-emitting diodes based on the "far-field plasmonic effect" of gold nanoparticles. J. Mater. Chem. C 8 6615-6622 (2020).

144. Liu, S. Y., Fang, X. H., Wang, Y. M. \& Zhang, X. P. Two-photon pumped amplified spontaneous emission based on all-inorganic perovskite nanocrystals embedded with gold nanorods. Opt. Mater. 81, 55-58 (2018).

145. Xu, Z. S., Liu, X. F., Qiu, J. R. \& Cheng, C. Enhanced luminescence of CsPbBr3 perovskite quantum-dot-doped borosilicate glasses with Ag nanoparticles. Opt. Lett. 44, 5626-5629 (2019).

146. Yun, J. H. et al. Enhanced luminescence of $\mathrm{CsPbBr} 3$ perovskite nanocrystals on stretchable templates with $\mathrm{Au} / \mathrm{SiO} 2$ plasmonic nanoparticles. Opt. Lett. 43, 2352-2355 (2018).

147. Li, H. M. et al. Polymer spacer tunable Purcell-enhanced spontaneous emission in perovskite quantum dots coupled to plasmonic nanowire networks. Phys. Chem. Chem. Phys. 21, 22831-22838 (2019).

148. Li, H. M. et al. Purcell-enhanced spontaneous emission from perovskite quantum dots coupled to plasmonic crystal. J. Phys. Chem. C 123, 25359-25365 (2019).

149. Juan, F. Y. et al. Photoluminescence enhancement of perovskite $\mathrm{CsPbBr}_{3}$ quantum dots by plasmonic Au nanorods. Chem. Phys. 530, 110627 (2020).

150. Shi, Z. F. et al. Localized surface plasmon enhanced all-inorganic perovskite quantum dot light-emitting diodes based on coaxial core/shell heterojunction architecture. Adv. Funct. Mater. 28, 1707031 (2018).

151. Chen, J. et al. Room temperature continuous-wave excited biexciton emission in perovskite nanoplatelets via plasmonic nonlinear fano resonance. Commun. Phys. 2, 80 (2019)

152. Adamo, G. et al. Metamaterial enhancement of metal-halide perovskite luminescence. Nano Lett. 20, 7906-7911 (2020)

153. Wang, S. et al. Maskless fabrication of aluminum nanoparticles for plasmonic enhancement of lead halide perovskite lasers. Adv. Opt. Mater. 5, 1700529 (2017).

154. Huang, C. et al. Formation of lead halide perovskite based plasmonic nanolasers and nanolaser arrays by tailoring the substrate. ACS Nano $\mathbf{1 2}$ 3865-3874 (2018).

155. Li, Y. J. et al. Output coupling of perovskite lasers from embedded nanoscale plasmonic waveguides. J. Am. Chem. Soc. 138, 2122-2125 (2016).

156. Wu, X. et al. Highly stable enhanced near-infrared amplified spontaneous emission in solution-processed perovskite films by employing polymer and gold nanorods. Nanoscale 11, 1959-1967 (2019).

157. Chen, F. et al. Detachable surface plasmon substrate to enhance $\mathrm{CH}_{3} \mathrm{NH}_{3} \mathrm{PbBr}_{3}$ lasing. Opt. Commun. 452, 400-404 (2019).

158. Hsieh, Y. H. et al. Perovskite quantum dot lasing in a gap-plasmon nanocavity with ultralow threshold. ACS Nano 14, 11670-11676 (2020).

159. Lu, Y. J. et al. Upconversion plasmonic lasing from an organolead trihalide perovskite nanocrystal with low threshold. ACS Photonics 8, 335-342 (2021).

160. Huang, Z. T. et al. Hybrid plasmonic surface lattice resonance perovskite lasers on silver nanoparticle arrays. Adv. Opt. Mater. 9 (2021)

161. Kao, T. S. et al. Localized surface plasmon for enhanced lasing performance in solution-processed perovskites. Opt. Express 24, 20696-20702 (2016).

162. Shang, Q. et al. Surface plasmon enhanced strong exciton-photon coupling in hybrid inorganic-organic perovskite nanowires. Nano Lett. 18, 3335-3343 (2018)
163. Yu, H. C. et al. Organic-inorganic perovskite plasmonic nanowire lasers with a low threshold and a good thermal stability. Nanoscale 8, 19536-19540 (2016).

164. Wu, Z. Y. et al. All-inorganic $\mathrm{CsPbBr}_{3}$ nanowire based plasmonic lasers. Adv Opt. Mater. 6, 1800674 (2018)

165. Yang, S. et al. Conference on Lasers and Electro-Optics. SM4l.1 (Optical Society of America, 2018).

166. Cong, H. L. et al. A smart magnetic responsive microfiltration membrane based on three-dimensionally inverse colloidal crystal. Integr. Ferroelectr. 206 112-121 (2020).

167. Dong, Y. H. et al. Improving all-inorganic perovskite photodetectors by preferred orientation and plasmonic effect. Small 12, 5622-5632 (2016).

168. Du, B. W. et al. Plasmonic-functionalized broadband perovskite photodetector. Adv. Opt. Mater. 6, 1701271 (2018).

169. Gu, Q. C. et al. Plasmon enhanced perovskite-metallic photodetectors. Mater Des. 198, 109374 (2021).

170. Sun, Z. H., Aigouy, L. \& Chen, Z. Y. Plasmonic-enhanced perovskite-graphene hybrid photodetectors. Nanoscale 8, 7377-7383 (2016).

171. Ghosh, J., Natu, G. \& Giri, P. K. Plasmonic hole-transport-layer enabled selfpowered hybrid perovskite photodetector using a modified perovskite deposition method in ambient air. Org. Electron. 71, 175-184 (2019).

172. Li, S. et al. Phosphorus-doped $\mathrm{Fe}_{3} \mathrm{O}_{4}$ nanoflowers grown on $3 \mathrm{D}$ porous graphene for robust $\mathrm{pH}-$ Universal hydrogen evolution reaction. Int. J. Hydrog. Energy 45, 4435-4443 (2020).

173. Li, M. Y. et al. Enhanced spatial light confinement of all inorganic perovskite photodetectors based on hybrid plasmonic nanostructures. Small 16 2004234 (2020).

174. Fang, Z. G., Wang, S. P., Kong, X. K. \& Liu, Q. C. Synthesis of the morphologycontrolled porous $\mathrm{Fe}_{3} \mathrm{O}_{4}$ nanorods with enhanced microwave absorption performance. J. Mater. Sci. -Mater. El. 31, 3996-4005 (2020).

175. La, J. A. et al. Highly sensitive and fast perovskite photodetector functionalized by plasmonic Au nanoparticles-alkanethiol assembly. Appl. Surf. Sci. $\mathbf{5 3 8}$ 148007 (2021).

176. Li, D. Y. et al. Plasmonic photonic crystals induced two-order fluorescence enhancement of blue perovskite nanocrystals and its application for highperformance flexible ultraviolet photodetectors. Adv. Funct. Mater. 28, 1804429 (2018).

177. Elshorbagy, M. H., Cuadrado, A. \& Alda, J. Narrow absorption in ITO-free perovskite solar cells for sensing applications analyzed through electromagnetic simulation. Appl, Sci. 9, 4850 (2019).

178. Qiao, X. Z., Xue, Z. J., Liu, L., Liu, K. Y. \& Wang, T. Superficial-layer-enhanced Raman scattering (SLERS) for depth detection of noncontact molecules. Adv Mater. 31, 1804275 (2019).

179. Zeng, S. W. et al. Plasmonic Metasensors Based on 2D Hybrid Atomically Thin Perovskite Nanomaterials. Nanomaterials 10, 1289 (2020).

180. Zhang, T. T. et al. Organic pollutant photodecomposition by $\mathrm{Ag} / \mathrm{KNbO}_{3}$ nanocomposites: a combined experimental and theoretical study. J. Phys. Chem. C. 120, 2777-2786 (2016).

181. Saris, S., Loiudice, A., Mensi, M. \& Buonsanti, R. Exploring energy transfer in a metal/perovskite nanocrystal antenna to drive photocatalysis. J. Phys. Chem. Lett. 10, 7797-7803 (2019).

182. Chanana, A. et al. Colour selective control of terahertz radiation using twodimensional hybrid organic inorganic lead-trihalide perovskites. Nat. Commun. 8, 1328 (2017).

183. Zhou, J. H. et al. Ultrasensitive polarization-dependent terahertz modulation in hybrid perovskites plasmon-induced transparency devices. Photonics Res. 7, 994-1002 (2019)

184. Zhou, Z. et al. From 1D to 3D: Tunable sub-10 nm gaps in large area devices. Adv. Mater. 28, 2956-2963 (2016)

185. Wang, Z. et al. Free-standing plasmonic chiral metamaterials with 3D resonance cavities. ACS Nano 12, 10914-10923 (2018).

186. Ai, B., Moehwald, H., Wang, D. \& Zhang, G. Advanced colloidal lithography beyond surface patterning. Adv. Mater. Interfaces 4, 1600271 (2017).

187. Jeon, N. J. et al. A fluorene-terminated hole-transporting material for highly efficient and stable perovskite solar cells. Nat. Energy 3, 682-689 (2018).

188. Tao, Q. L., Xu, P. C., Li, M. J. \& Lu, W. C. Machine learning for perovskite materials design and discovery. Npj Comput. Mater. 7, 1-18 (2021).

189. Saidi, W. A., Shadid, W. \& Castelli, I. E. Machine-learning structural and electronic properties of metal halide perovskites using a hierarchical convolutional neural network. Npj Comput. Mater. 6, 36 (2020).

190. So, S., Badloe, T., Noh, J., Rho, J. \& Bravo-Abad, J. Deep learning enabled inverse design in nanophotonics. Nanophotonics 9, 1041-1057 (2020). 
191. Sajedian, I., Lee, H. \& Rho, J. Design of high transmission color filters for solar cells directed by deep Q-learning. Sol. Energy 195, 670-676 (2020).

192. Sajedian, I., Badloe, T., Lee, H. \& Rho, J. Deep Q-network to produce polarization-independent perfect solar absorbers: a statistical report. Nano Convergence 7, 26 (2020).

193. Zhang, W. et al. Enhancement of perovskite-based solar cells employing core-shell metal nanoparticles. Nano Lett. 13, 4505-4510 (2013).

194. Hsu, H. L. et al. Enhanced efficiency of organic and perovskite photovoltaics from shape-dependent broadband plasmonic effects of silver nanoplates. Sol. Energy Mater. Sol. Cells 140, 224-231 (2015).

195. Lu, Z. L. et al. Plasmonic-enhanced perovskite solar cells using alloy popcorn nanoparticles. Rsc Adv. 5, 11175-11179 (2015).

196. Nourolahi, H., Behjat, A., Zarch, S. M. M. H. \& Bolorizadeh, M. A. Silver nanoparticle plasmonic effects on hole-transport material-free mesoporous heterojunction perovskite solar cells. Sol. Energy 139, 475-483 (2016).

197. Yu, H., Roh, J., Yun, J. \& Jang, J. Synergistic effects of three-dimensional orchid-like TiO2 nanowire networks and plasmonic nanoparticles for highly efficient mesoscopic perovskite solar cells. J. Mater. Chem. A 4, 7322-7329 (2016)

198. Wang, D. et al. Interfacial engineering of hole transport layers with metal and dielectric nanoparticles for efficient perovskite solar cells. Phys. Chem. Chem. Phys. 19, 25016-25024 (2017)

199. Zarick, H. F. et al. Ultrafast carrier dynamics in bimetallic nanostructureenhanced methylammonium lead bromide perovskites. Nanoscale 9 , 1475-1483 (2017).

200. Zhang, X. M. et al. Performances enhancement in perovskite solar cells by incorporating plasmonic Au NRs@SiO 2 at absorber/HTL interface. Sol. RRL 1, 1700151 (2017).

201. Dong, $\mathrm{H}$. et al. Plasmonic enhancement for high efficient and stable perovskite solar cells by employing "hot spots" Au nanobipyramids. Org. Electron. 60, 1-8 (2018).

202. Que, M. D. et al. Tunable plasmon-enhanced broadband light harvesting for perovskite solar cells. J. Power Sources 383, 42-49 (2018).
203. Zhao, W. et al. Enhanced efficiency and stability of planar perovskite solar cells using a dual electron transport layer of gold nanoparticles embedded in anatase TiO2 films. ACS Appl. Energy Mater. 3, 9568-9575 (2020).

204. Bi, W. B. et al. Dye sensitization and local surface plasmon resonanceenhanced upconversion luminescence for efficient perovskite solar cells. ACS Appl. Mater. Interfaces 12, 24737-24746 (2020).

205. Qin, P. L. et al. Grain boundary and interface passivation with core-shell Au@CdS nanospheres for high-efficiency perovskite solar cells. Adv. Funct. Mater. 30, 1908408 (2020).

206. Li, H., Zhou, S. J. \& Yin, L. W. Surface plasmon resonance effect enhanced $\mathrm{CsPBr}_{3}$ inverse opals for high-performance inorganic perovskite solar cells. Adv. Mater. Interfaces 7, 1901885 (2020).

207. Cui, X. et al. Tailoring carrier dynamics in perovskite solar cells via precise dimension and architecture control and interfacial positioning of plasmonic nanoparticles. Energy Environ. Sci. 13, 1743-1752 (2020).

208. Ghosh, A. et al. Core/shell nanocrystal tailored carrier dynamics in hysteresisless perovskite solar cells with similar to $20 \%$ efficiency and long operational stability. J. Phys. Chem. Lett. 11, 591-600 (2020).

209. Kesavan, A. V., Rao, A. D. \& Ramamurthy, P. C. Tailoring optoelectronic properties of $\mathrm{CH}_{3} \mathrm{NH}_{3} \mathrm{Pbl}_{3}$ perovskite photovoltaics using al nanoparticle modified $\mathrm{PC}_{61} \mathrm{BM}$ layer. Sol. Energy 201, 621-627 (2020).

210. Liu, S. et al. Synergy of plasmonic silver nanorod and water for enhanced planar perovskite photovoltaic devices. Sol. RRL 4, 1900231 (2020).

211. Lin, Y. T., Kumar, G. \& Chen, F. C. Interfacial plasmonic effects of gold nanoparticle-decorated graphene oxides on the performance of perovskite photovoltaic devices. Sol. Energy 211, 822-830 (2020).

212. Juan, F. Y. et al. Plasmonic Au Nanooctahedrons Enhance Light Harvesting and Photocarrier Extraction in Perovskite Solar Cell. ACS Appl. Energy Mater. 4, 3201-3209 (2021).

213. Stranks, S. D., Hoye, R. L. Z., Di, D. W., Friend, R. H. \& Deschler, F. The physics of light emission in halide perovskite devices. Adv. Mater. 31, 1803336 (2019).

214. Zeng, S. W., Liang, G. Z., Gheno, A., Vedraine, S. \& Yu, N. F. 2D perovskite-based metasurfaces for enhanced plasmonic sensing. Conf. Laser Electr. (2019). 